\title{
Structure in the First Quadrant of the Galaxy: an Analysis of "TMGS" Star Counts using the "SKY" Model
}

\author{
P. L. Hammersley ${ }^{1}$, M. Cohen $^{3}$, F. Garzón ${ }^{1,2}$, T. Mahoney ${ }^{1}$, M. López-Corredoira ${ }^{1}$ \\ ${ }^{1}$ Instituto de Astrofísica de Canarias, E-38200 La Laguna, Tenerife, Spain \\ 2 Departamento de Astrofísica, Universidad de La Laguna, La Laguna, Tenerife, Spain \\ ${ }^{3}$ Radio Astronomy Laboratory, 601 Campbell Hall, University of California, Berkeley, CA 94720
}

' received date : accepted date

\begin{abstract}
We analyse the stellar content of almost $300 \mathrm{deg}^{2}$ of the sky close to the Galactic plane by directly comparing the predictions of the SKY model (Cohen 1994,1995; Wainscoat et al. 1992) with star counts taken from the Two Micron Galactic plane Survey (TMGS: Garzón et al. 1993). Through these comparisons we can examine discrepancies between counts and model and thereby elicit an understanding of Galactic structure. Over the vast majority of areas in which we have compared the TMGS data with SKY's predictions we find very good accord; so good that we are able to remove the disc source counts to highlight structure in the plane. The exponential disc is usually dominant, but by relying on SKY's predicted disc counts we have been able to probe the molecular ring, spiral arms, and parts of the bulge. The latter is clearly triaxial. We recognize a number of off-plane dust clouds not readily included in models. However, we find that, whilst the simple exponential extinction function works well in the outer Galaxy, closer than about $4 \mathrm{kpc}$ to the Centre the extinction drops dramatically. We also examine the shape of the luminosity function of the bulge and argue that the cores of all spiral arms we have observed contain a significant population of supergiants that provides an excess of bright source counts over those of a simple model of the arms. Analysis of one relatively isolated cut through an arm near longitude 65 degrees categorically precludes any possibility of a $\operatorname{sech}^{2} z$ stellar density function for the disc.
\end{abstract}

Key words: Galaxy: structure, stellar content - Infrared: stars

\section{INTRODUCTION}

Although star counts have long been used for the determination of Galactic structure (see Paul 1993), there are still a large number of parameters which are not well determined. Reviews of star count surveys can be found in Bahcall (1986), Majewski (1993) and Reid (1993). Many visible studies have been directed towards the Galactic poles where extinction in the Galactic plane can largely be ignored. Star counts, velocity distributions and metallicity have been used to determine and separate the various Galactic populations, such as the old disc, young disc and halo. Detailed reviews are given, for example, by Gilmore, Wyse \& Kuijken (1989) and Freeman (1987), and references therein. Hence, the vertical structure of the local Galaxy is well measured (although there remain contentious issues such as the thick disc). The horizontal structures are less well studied. Robin, Crézé \& Mohan (1992) obtained deep visible star counts in the anticentre region and concluded that the disc has a scale length of $2.5 \mathrm{kpc}$ and a radial cutoff at about $14 \mathrm{kpc}$. However, apart from individual clear windows, visible star counts in the plane are limited to a maximum heliocentric distance of about $3 \mathrm{kpc}$ (Schmidt-Kaler 1977), because of the high extinction. A method of overcoming this, at least in part, is to observe in the infrared, where the extinction is significantly lower. Infrared source counts detect sources to a greater distance and are far less affected by local dust, so the measured distribution of sources is closer to the true distribution.

To date, infrared surveys of the Galactic plane capable of detecting point sources have been limited. The largest IR survey to date was that performed by the Infrared Astronomical Satellite, IRAS, which mapped nearly the entire sky at 12, 25, 60 and $100 \mu \mathrm{m}$. Much work on Galactic structure has been enabled by IRAS particularly on the distribution of very late type stars (see Beichman, 1987, for a review). However, at large distances, IRAS could detect only extremely luminous sources (e.g. OH/IR stars) and was severely confusion-limited over much of the Galactic 
Table 1. Principal attributes of SKY. For more details see WCVWS, Cohen (1994,1995), and Cohen et al. (1994).

\begin{tabular}{cccccc}
\hline \hline $\begin{array}{c}\text { Geometric } \\
\text { components }\end{array}$ & $\begin{array}{c}\text { No. of types } \\
\text { of source }\end{array}$ & $\begin{array}{c}\text { Radial scale } \\
\text { length }\end{array}$ & $\begin{array}{c}\text { Source scale } \\
\text { height }\end{array}$ & $\begin{array}{c}\text { Mag. range } \\
\text { modelled }\end{array}$ & $\begin{array}{c}\text { Wavelength } \\
\text { range }\end{array}$ \\
\hline disc; bulge; arms, spurs, Gould's Belt; ring; halo & 87 & $3.5 \mathrm{kpc}$ & $90-325 \mathrm{pc}$ & $(-10,+30)$ & $0.14-35 \mu \mathrm{m}$ \\
\hline \hline
\end{tabular}

Table 2. Summary of regions of the sky analysed. Listed for each strip are the declination and $l$ as the strip crosses the GP. Normally a strip is identified by one of these two parameters. Also listed are the starting and ending points for each strip, the number of useful areas of comparison with SKY, the area per bin, and the relevant figures in the Appendix for this strip. The area is important as it relates observed TMGS counts in an area to counts per $\operatorname{deg}^{2}$ (and the associated Poisson errors are calculable as shown in Figure 1).

\begin{tabular}{ccccccc}
\hline \hline $\begin{array}{c}\text { Dec. } \\
(\mathrm{J} 2000)\end{array}$ & $\begin{array}{c}\text { Plane crossing } \\
l\end{array}$ & $\begin{array}{c}\text { Start } \\
(l, b)\end{array}$ & $\begin{array}{c}\text { End } \\
(l, b)\end{array}$ & $\begin{array}{c}\text { No. useful } \\
\text { zones }\end{array}$ & $\begin{array}{c}\text { Typical } \\
\text { area }\left(\mathrm{deg}^{2}\right)\end{array}$ & Fig. No. \\
\hline-30 & -1 & $(6.54,-14.5)$ & $(-5.53,7.5)$ & 45 & 1.9 & A1,B1, C1,D1 \\
-22 & 7 & $(15.007,-15.0)$ & $(-2.223,14.5)$ & 61 & 2.0 & A2,B2,C2,D2 \\
-11 & 21 & $(27.495,-15.0)$ & $(11.619,14.5)$ & 61 & 0.69 & A3,B3,C3,D3 \\
-5 & 27 & $(29.294,-4.5)$ & $(24.124,5.5)$ & 20 & 0.18 & A4,B4,C4,D4 \\
-1 & 31 & $(38.994,-15.0)$ & $(23.264,15.0)$ & 61 & 2.7 & A5,B5,C5,D5 \\
+3 & 37 & $(39.74,-5.5)$ & $(33.968,6.0)$ & 24 & 0.25 & A6,B6,D6 \\
+19 & 53 & $(56.215,-5.0)$ & $(50.582,5.5)$ & 22 & 0.18 & A6,B7,D7 \\
+27 & 65 & $(73.688,-5.5)$ & $(66.817,5.5)$ & 61 & 2.0 & A7,B8,C6,D8 \\
+32 & 70 & $(75.837,-15.0)$ & $(56.995,15.5)$ & 60 & 0.56 & B9,D9 \\
\hline \hline
\end{tabular}

plane. The Two Micron Sky Survey (Neugebauer \& Leighton 1969: hereafter TMSS) also had a low limiting magnitude $\left(m_{K} \approx 3\right)$ and so could not detect sources in the inner Galaxy. Other surveys with greater sensitivity have tended to be restricted to the Galactic Centre (hereafter GC) region. Those that have, at least in part, examined the disc (e.g. Eaton et al. 1984; Kawara et al. 1982) have covered only very small areas of sky, typically a few hundred $\operatorname{arcmin}^{2}$. These surveys ran the risk of sampling an area with unusually high extinction, as well as suffering from low source counts, and were unable to show the large-scale distribution of sources.

Garzón et al. (1993) have described the Two Micron Galactic Survey (hereafter TMGS). This is a $K$-band survey with an approximate limit of $m_{K} \sim 10$ which represents a valuable addition to our knowledge of the near-infrared Galaxy, deepening the broad sweep coverage of the plane by a factor of almost 1000 compared with the TMSS. It fulfils an important transitional role, providing a homogeneous data base over a number of regions in the Galactic plane at a time when both the hemispheric DENIS (Epchtein 1998) and all-sky 2MASS (Skrutskie 1998) three-band surveys are in routine operation. These new surveys will go about $4-5$ magnitudes fainter than TMGS and offer simultaneous observations in $I J K^{\prime}$ or $J H K^{\prime}$. Nevertheless, a $K$-band survey with a magnitude limit of $m_{K} \sim 10$ such as the TMGS can usefully probe the structure of our Galaxy.

Hammersley et al. (1994) and Calbet et al. (1995) explored the morphology of star counts from the TMGS by heuristic methods, while Hammersley et al. (1995) deduced the displacement of the Sun from the plane from the slight north-south asymmetry of counts crossing the plane. However, these papers are based principally in looking at the very obvious features seen in the the TMGS. In order to understand the $K$ star counts more fully, a detailed model is required which allows the total counts to be broken down into counts from the various Galactic geometric components in each magnitude range. The model that we have chosen to work with is described by Cohen (1994), who has enhanced the "SKY" model for the point source sky originally described by Wainscoat et al. (1992: hereafter WCVWS), adding molecular clouds for mid-infrared realism, extending the Galactic structure to model the far-ultraviolet sky, and displacing the Sun 15 pc north of the plane (Cohen 1995).

The purpose of this paper is to describe our use of the SKY model to provide a formal basis for interpreting the TMGS source counts. Our eventual objective is to refine the model, guided by any discrepancies that we find with the TMGS data base. To do this we contrast the TMGS data from almost 600 patches of sky (some $296 \mathrm{deg}^{2}$ ) in the vicinity of the plane with the predictions of the SKY model, to investigate the stellar content and probable geometry of the Galactic plane. The advantage of this approach is that we can emphasize otherwise slight deviations from expectation, and thereby define those regions in which the TMGS has uncovered substantive evidence of structure contrary to that in a formal model. We present analyses with SKY of TMGS star counts along entire survey strips, from $10^{\circ}$ to $30^{\circ}$ in length, cutting the plane at a variety of longitudes. We conclude that SKY provides a very good overall description of $K$ counts in the plane, which is chiefly dominated by the exponential disc, but that the TMGS offers important insights into the character of structure very close to the Galactic plane and the nature of the bulge. We find four basic styles of statistically significant deviation of SKY's predictions from the observed counts. One appears to arise from a significant constituent of the ring, or arm, populations not currently in SKY, and strongly confined in latitude close to the plane in the inner Galaxy (longitudes $20^{\circ}-31^{\circ}$ ). A second type of behaviour suggests that the luminosity function (hereafter LF) of the inner bulge is not that implicitly rep- 
Table 3. Summary of lines-of-sight covered. Listed for each strip is the closest approach to the GC (assuming $\mathrm{R}_{\odot}=8500 \mathrm{pc}$ ) and the features that are sampled. The question marks with the ring/bar depend on the interpretation of the counts (see Sections 8,9). The Arm crossings listed are those closest to the sun (Sct=Scutum, Sgr=Sagittarius, and Per=Perseus arm). The * indicates that the line of sight is almost tangential to the spiral arm.

\begin{tabular}{cccccccc}
\hline \hline Dec. & $\begin{array}{c}\text { Plane crossing } \\
l\end{array}$ & $\begin{array}{c}\text { Closest distance } \\
\text { to the GC }(\mathrm{pc})\end{array}$ & Bulge & Ring/bar & Sct & Sgr & Per \\
\hline-30 & -1 & 150 & $\mathrm{Y}$ & $?$ & $\mathrm{Y}$ & $\mathrm{Y}$ & \\
-22 & 7 & 1050 & $\mathrm{Y}$ & $?$ & $\mathrm{Y}$ & $\mathrm{Y}$ & \\
-11 & 21 & 3050 & & $\mathrm{Y}$ & $\mathrm{Y}$ & $\mathrm{Y}$ & \\
-5 & 27 & 3850 & & $\mathrm{Y}$ & $\mathrm{Y}$ & $\mathrm{Y}$ & \\
-1 & 31 & 4400 & & $?$ & $\mathrm{Y}^{*}$ & $\mathrm{Y}$ & \\
+3 & 37 & 5100 & & & & $\mathrm{Y}$ & \\
+19 & 53 & 6800 & & & & $\mathrm{Y}^{*}$ & \\
+27 & 65 & 7700 & & & & & $\mathrm{Y}$ \\
+32 & 70 & 8000 & & & & & $\mathrm{Y}$ \\
\hline \hline
\end{tabular}

resented by SKY. The third set of departures from an ideal model is caused by spiral arms that do not lie in the plane (cf. WCVWS; Cohen 1994). The remaining type of pattern is due both to off-plane dark clouds not included in SKY's smooth analytic expression of the extinction, and lines of sight through the Galaxy which have unusually high or low distributed (rather than localized) extinction.

\section{THE TMGS AND THE SKY MODEL}

\subsection{The TMGS}

The TMGS (Garzón et al. 1993) is a collaborative project between the Instituto de Astrofísica de Canarias, Tenerife, and Imperial College, London, to map large parts of the Galactic bulge and plane. Observations were made between 1988 and 1995 on the 1.5-m Carlos Sánchez Telescope of the Observatorio del Teide, Tenerife. Some $350 \mathrm{deg}^{2}$ of sky have been mapped down to about 10 mag in $K$, resulting in a data set of about 700,000 sources. The main areas are in strips about $30^{\circ}$ in RA (centred on the Galactic plane) by about $1.5^{\circ}$ in declination. Most TMGS sources lie within a few degrees of the Galactic plane, the majority having no optical counterparts to at least $m_{V}=16$, implying that they are sources deep within the Galaxy. The star counts presented in this paper were made using all of the data in each area. Although the data were taken in strips in RA, the TMGS catalogue has been converted to Galactic coordinates and the counts made using $1^{\circ}$ bins in latitude.

\subsection{SKY: the Model}

The original SKY model described in WCVWS incorporates six fully detailed Galactic components: an exponential disc; a Bahcall-type bulge; a de Vaucouleurs halo; a 4-armed spiral with 2 local spurs; Gould's Belt and a circular molecular ring. Each geometrical element may contain up to 87 source categories, derived from detailed analyses of the content of the IRAS sky (Walker et al. 1989). These comprise 33 "normal" stellar types; 42 types of AGB star, both oxygen- and carbon-rich; 6 types of object that are distinct from others only in their MIR high luminosity; and 6 types of exotica including H II regions, planetaries, and reflection nebulae.
Every category has its own set of absolute magnitudes and dispersion in ten hardwired passbands, and an individual scale height and volume density in the local solar neighbourhood. Some sources are absent from some components (the arms and ring are made rich in high-mass stars; the halo is deficient in these).

We have used version 4 of SKY as detailed by Cohen $(1994,1995)$ and Cohen et al. (1994), the principal attributes are summarized in Table 1. This is different from WCVWS in that it has the solar offset from the plane and reduced halo:disc ratio, however, we have not included the additional molecular cloud populations essential to obtain a high-fidelity representation of the mid-infrared sky. This omission is justified because the clouds were inserted by Cohen (1994) for IRAS wavelengths of 12 and $25 \mu \mathrm{m}$, which are essentially insensitive to extinction. Therefore only the extra sources were included and not the extra extinction through the clouds, yet the extra populations associated with these clouds are surely accompanied by extra extinction not accounted for within SKY. Cohen et al. (1994) demonstrated in the far-ultraviolet that one could assign a value to the additional extinction encountered through local molecular clouds using SKY. Within the TMGS archive one can clearly recognize obscuring clouds beyond which no $2-\mu \mathrm{m}$ sources are detected, although at $K$ only the dense cores cause a significant effect on the counts and so the effect of the extinction is generally limited to single TMGS zones. Consequently, without a detailed prior knowledge of the additional extinction along the specific TMGS lines of sight, it is preferable to model without including these molecular complexes at wavelengths susceptible to appreciable attenuation through clouds of $A_{V}>10$ mag. In this way one can more readily identify those lines of sight that intercept dark clouds at $K$. Furthermore, the IRAS sources detected in the clouds had warm dust which makes the sources very luminous at $12 \mu \mathrm{m}$. This warm dust does not have as large an effect on the absolute magnitudes at $2.2 \mu \mathrm{m}$, so the sources will be detected at $K$ but will not be that much brighter than the sources without warm dust. Hence they will be more difficult to see against the background of disc or bulge sources. This is the sole modification made to the published description of SKY4. 
P. L. Hammersley et al.

\subsection{Why Confront SKY and the TMGS?}

The SKY model was originally built to reproduce the IRAS $12-$ and $25-\mu \mathrm{m}$ source counts. However, it has been successfully compared with observations at many other wavelengths including the far-ultraviolet (Cohen 1994; Cohen et al. 1994). The model has already been compared with DENIS $K$ star counts in small regions (Ruphy et al. 1997) for the magnitude range $m_{K}=10-13$. However, the comparison with the TMGS is possibly the most challenging for the model because its magnitude range, $m_{K}=5-9$, probes the brightest sources in the LFs of the geometric components such as the arms and bulge. At $m_{K}$, these LFs are largely unknown, particularly for the arms, but the contrast with the disc will be maximal so any discrepancy with the model will readily be seen. Further, the areas to be studied are long cuts through the arms and disc, providing nearby reference regions out of the arms. This, too, allows differences to be explored in a manner that is impossible given only a single region. The final important advantage is the relatively large area of sky covered which, in many regions, affords meaningful star counts to be produced as bright as $m_{K}=5$.

The fact that SKY produces good matches to IRAS (Cohen 1995) and visible stars counts towards the NGP (WCVWS) does not guarantee that it will successfully match the TMGS star counts. The $K$ LF is dominated by photospheres whereas, for IRAS, the principal populations detected at significant distances are all dust-enshrouded. Clearly, the IRAS sources will be detected by the TMGS but are many magnitudes fainter, greatly diminishing their importance. Thus, whilst SKY may get the LF of the bulge correct for $I R A S$, this does not necessarily imply correctness for the TMGS. Garzón et al. (1993) already noted that the Galaxy at $2.2 \mu \mathrm{m}$ looks very different from that in the visible because of extinction in the plane. Furthermore, the principal sources detected by the TMGS are K4-M0 giants or M-supergiants, which are far less important in visible star counts when coupled with extinction.

\section{CONFRONTATION OF THE MODEL BY THE OBSERVATIONS}

In the Appendix (Figures A1-A7, D1-D9), we present TMGS counts along nine strips in the first quadrant. These illustrate our interpretations of the counts by direct comparisons with the predictions of SKY. Differential counts were scaled per square degree per magnitude, using entirely independent TMGS data in 0.2-mag wide bins.

Table 2 summarizes for each strip: the J2000 declination; the longitude of the Galactic plane crossing; the bounding coordinates; the number of useful (i.e. with tolerable implicit Poisson errors) zones we present in the relevant figure; the typical area sampled at each position along the strip (this varies slightly along a strip); and the figure which illustrates the star counts and SKY predictions. Table 3 amplifies this information by indicating the closest approach of each line of sight to the Galactic Centre and which (non-disc) geometric components contribute to the predicted counts.

Our analyses depend on three basic analytical tools: differential star counts (Figures A1-A7, D1-D9); profiles of cumulative counts in a specific $K$-magnitude bin as a function of latitude along each strip (Figures B1-B9); and similar profiles created after subtracting the disc component predicted by SKY from the observed counts (Figures C1-C9). In both Figures $\mathrm{B}$ and $\mathrm{C}$, we represent the observations by solid lines and the model counts by dashed lines.

Figures 1(a)-(h) present eight representative fields, taken from the montages shown in the Appendix, for which we explicitly plot the Poisson error bars appropriate to the original counts (which were scaled by 5 , to yield counts per mag from the 0.2-mag binned data, and again, according to the actual area sampled, to yield counts per $\operatorname{deg}^{2}$ ). Figure 1 distinguishes the counts arising from separate geometric components as follows: solid line - total count; dotted or less heavy line - disc; long dashes - spiral arms, local spurs and Gould's Belt; short dashes - bulge; short dashes and dots - molecular ring; long dashes and dots - halo. The figures were chosen to be representative of the various regions covered as well as showing specific features in their form which are discussed later in the paper.

As with all surveys, Malmquist bias can arise in TMGS and we see its effects in Figure $1(\mathrm{~h})$ where the counts in the final few faint bins rise abruptly before the limit of completeness is attained. Note also that this is a region in which the actual source density is relatively low. In general, the direct comparison of SKY and TMGS counts indicates that TMGS is complete to a magnitude between 9.2 and 9.8, depending on the local surface density of sources.

\section{EXTINCTION}

\subsection{Off-Plane Dark Clouds}

As with all previous versions of the model, SKY4 represents interstellar extinction simply by the product of two exponentials; one radial, with scale length of $3.5 \mathrm{kpc}$ (matching that of the stars); one vertical, with scale height of $100 \mathrm{pc}$ (matching that of the youngest stars still associated with dark clouds). This version of SKY does not include off-plane dark clouds so, if a TMGS strip runs through the core of such a cloud, the counts will be significantly decreased when compared with the model (however, the greatly reduced extinction at $K$ compared with the visible implies it requires many magnitudes of visible extinction to cause a significant effect on the TMGS counts). Figures A5,B5,D5 illustrates this phenomenon for the area centred on $l=29.3^{\circ}, b=4^{\circ}$ from the $l=31^{\circ}$ strip. One sees that the TMGS counts fall well below the predictions of SKY yet, at $b=2.5^{\circ}$ or $b=5^{\circ}$, the model and the TMGS are in good agreement. Figure 1(a) enlarges the plot for $b=4^{\circ}$, the deepest discrepancy, and adds the Poisson error bars. This location corresponds to a known off-plane cloud which clearly shows up in emission in longer-wavelength $C O B E$ maps, whereas the area is almost devoid of visible stars. In fact, the core of this cloud does not fill the TMGS bin at this location and sampling at higher resolution would decrease the TMGS counts even more at the centre of the cloud. The only potential H I counterpart (Weaver 1998, priv. com.) is a fairly uniformly dark patch (i.e. an absence of $\mathrm{H} \mathrm{I}$ ) well-centred in an $\mathrm{H}$ I emission ring, at an LSR velocity of $-15 \mathrm{~km} \mathrm{~s}^{-1}$, and about $1-1.5^{\circ}$ in diameter. Figure 1(b) shows another example, this time of a more localized off-plane cloud, whose influence is recognizable by an abrupt change in the observed source counts 

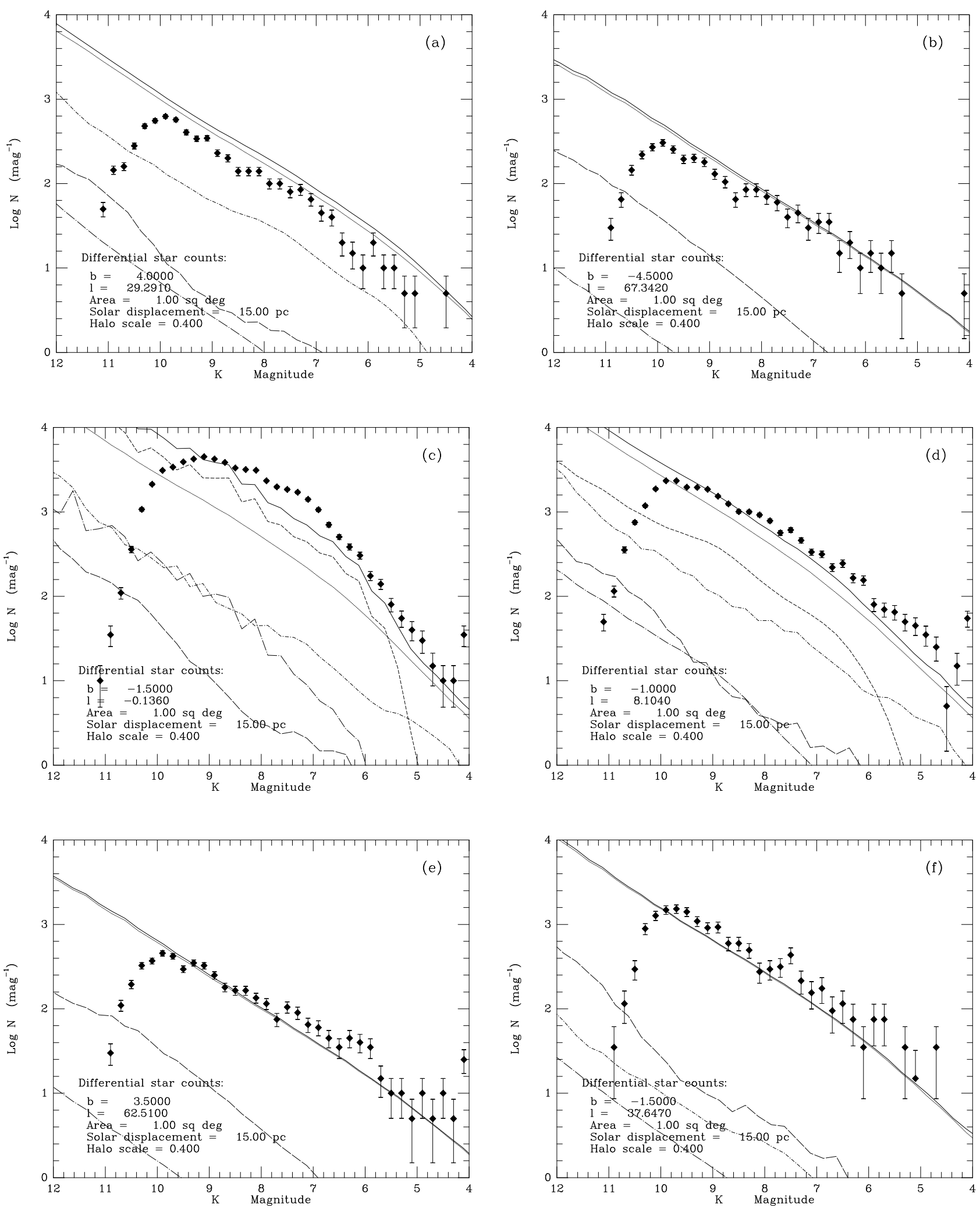

Figure 1:a-f 

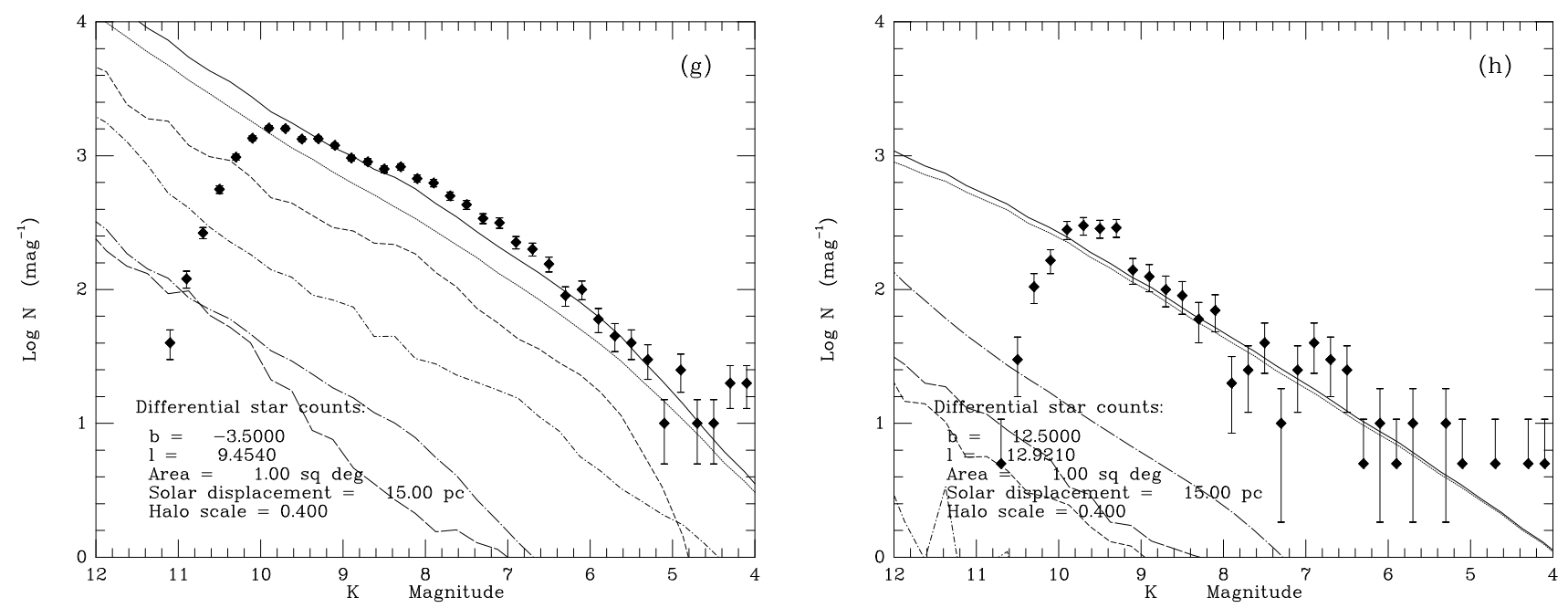

Figure 1. g-h. Differential star counts versus magnitude showing the detailed comparisons between the TMGS and SKY for sample regions. The black diamonds are the TMGS points with Poisson error bars marked. The SKY geometric components are marked as follows: solid line - total count; dotted or less heavy line - disc; long dashes - spiral arms, local spurs and Gould's Belt; short dashes bulge; short dashes and dots - molecular ring; long dashes and dots - halo

fainter than a specific magnitude. Here one sees an offset in source counts by 0.2 dex for $m_{K}>8.5$. Analysis of this line of sight by SKY indicates that the dominant contributors at this magnitude are disc K0-3 giants, at a probable distance of about $700 \mathrm{pc}$. Consequently, we suggest that one encounters a cloud with incremental $A_{V} \sim 5$ mag in this direction and at this distance. Again, H I maps (Weaver 1998, priv. comm.) show a sizeable void, bigger than that seen above (about $2^{\circ}$ in diameter), well-centred on this TMGS field, in an emission ring at an LSR velocity of $+38 \mathrm{~km} / \mathrm{s}$. In both cases, the presence of $\mathrm{H}$ I emission with central voids suggests that the hydrogen in these clouds is largely molecular rather than atomic.

\subsection{On-Plane Dark Clouds and General Extinction in the Plane}

The TMGS reveals a number of "on-plane" $\left(|b|<2^{\circ}\right)$ dark clouds, particularly towards the inner Galaxy. Hammersley et al. (1996) discussed the dark clouds which cause the major loss of stars at $l=-1^{\circ}, b=0^{\circ}$, and showed that most of the extinction comes from individual dark clouds close to the GC. However, there are individual clouds on, or very near, the plane in many TMGS areas which locally cause a noticeable loss of counts $\left(l=7^{\circ}, b=1^{\circ}\right.$ or $l=31^{\circ}, b=1^{\circ}$ : Figures A2, B2, D2, A5, B5 and B5)

SKY includes only the general extinction along the line of sight. This is modeled as a double exponential which continues all the way into the GC but is normalized in the solar neighbourhood. Figures B1- B9 show the cumulative TMGS and SKY star counts (the latter separated into total and disc contributions) for various strips, as a function of Galactic latitude, and the ratios of these totals at different limiting magnitudes. The effect of extinction on SKY's counts is clearly visible in these cuts as a dip in counts at $b=0^{\circ}$. For lines of sight with $l>30^{\circ}$, the dip on the plane is small, a few percent of the total counts, and there is very good agreement with the TMGS star counts. In the inner Galaxy, however, SKY predicts a major loss of counts on the plane, principally due to extinction in front of the bulge sources (the difference between SKY's total and disc curves for $m_{K}>7$ and the $l=7^{\circ}$ and $-1^{\circ}$ strips is almost totally due to the bulge). The model does reasonably well near $l=-1^{\circ}, b=0^{\circ}$ although, as already stated, most of the extinction appears to come from identifiable dark clouds close to the GC, not represented in SKY. However, SKY substantially over-predicts the extinction on the plane at $l=7^{\circ}$, $b=0^{\circ}$. The ratios of model-to-observed star counts for this region show a narrow peak which the original plot indicates is due to extra extinction in SKY.

Hammersley et al. (1998) show that the extinction at the radius of the molecular $\operatorname{ring}\left(0.4 R_{\odot}<R<0.5 R_{\odot}\right)$ is about a factor of three above that predicted by a simple exponential model yet SKY's analytical function for the extinction in the outer Galaxy (e.g. $\mathrm{R}>0.5 \mathrm{R} \odot$ ) gives a reasonable representation for the distributed extinction in the plane. Therefore, the only explanation for why SKY so significantly overestimates the extinction in the line of sight towards $l=7^{\circ}, b=0^{\circ}$, is that the extinction for $R>0.4 R_{\odot}$ drops to almost zero until a few hundred pc from the GC, where the features associated with the Centre begin (e.g. the molecular disc). The idea of there being a hole in the extinction in the inner Galaxy is not new: the CO maps clearly show a significant fall-off in density inside the molecular ring (Clemens, Sanders \& Scoville 1988; Combes 1991). Freudenreich (1998) also needed a hole in the extinction in the inner Galaxy to match the DIRBE surface brightness maps.

\section{THE EXPONENTIAL DISC FOR $B>5^{\circ}$}

SKY incorporates a single exponential disc with 87 categories of point source, each with its own scale height, colours, 
and local space density. The scale length of all sources is set to $3.5 \mathrm{kpc}$. SKY does not include a thick disc for several reasons. Firstly, it provides excellent fits to counts at a wide variety of wavelengths without using a thick disc. Secondly, the proportion of disc counts contributed by the thick disc, even at optical wavelengths where metallicities and kinematics may help separate these two populations, is minimal ( $<2 \%$; e.g. Gilmore, Wyse \& Kuijken 1989). Thirdly, the stars of the thick disc contribute negligibly in the infrared, far below the levels of Poisson errors of the TMGS (and many other) data sets.

SKY separates young and old components by assigning the young population (OBA dwarfs and supergiants) a significantly smaller scale height, $90 \mathrm{pc}$, than the $325 \mathrm{pc}$ of the old populations. Hence, the vast majority of the sources that SKY predicts that the TMGS should see in the exponential disc are the old K- and M-giants for regions well away from the bulge and more than $5^{\circ}$ from the plane. The relevant figures are those for the strips at $l=21^{\circ}, 31^{\circ}$ and $65^{\circ}$ (Figures A3, A5, A7, D3, D5 and D8). Figures B3, B5 and B8 show the associated cumulative counts for the TMGS and the model for these regions, to $m_{K}=9$.

Both the form of the curves and the differential star counts show that SKY gives a good fit to the TMGS at all magnitudes in these longitudes, for $|b|>5^{\circ}$.

In most areas of the sky, the disc dominates the star counts, making the remaining geometric components more difficult to identify and analyse. However, the accuracy of the fit of SKY'S disc to the TMGS counts makes it entirely reasonable to subtract the model's disc counts from the TMGS counts. This highlights the distribution of sources in the arms, ring, and bulge, if accomplished with care.

Alternatively, some argue that an exponential disc is no longer justifiable at the lowest latitudes in the Galaxy, and that the $\operatorname{sech}^{2} z$ form might be physically more defensible. There are a number of papers comparing the $\operatorname{sech}^{2} z$ and exponential in the discs of external galaxies (e.g. van de Kruit 1988; De Grijs, Peletier \& van de Kruit 1997). However, the results, even in the infrared, are complicated by extinction when close to the plane of the galaxy. The $\operatorname{sech}^{2} z$ function asymptotically approaches an exponential as stellar densities fall far from the plane but, for latitudes within about $10^{\circ}$ of the plane, it fails utterly to reproduce the observed counts. We illustrate this failure in Figure 2. The $l=65^{\circ}$ strip is one in which we see only disc and arms, the latter falling almost two orders of magnitude below the counts from the former. Thus, it is an ideal location to experiment with a $\operatorname{sech}^{2} z$ distribution function. Figure 2 shows the cut through the plane at $m_{K}=9$ for two model discs for $l=65^{\circ}$, $|b| \leq 15^{\circ}$, one is exponential in height (this is a simplified model based on SKY but it is not the SKY model) and the second is identical except that it has a $\operatorname{sech}^{2} z$ height distribution. We have normalized the $\operatorname{sech}^{2} z$ curve to the wings of the cumulative latitude profile in the regions with $|b|>10^{\circ}$ as both functions work well, away from the plane. Yet, for all inner latitudes, the $\operatorname{sech}^{2} z$ function delivers no more than half the requisite counts. This is equivalent to saying that, everywhere in the disc, even in those locations where spiral arms and other components do not make appreciable contributions to counts, more than $50 \%$ of the observed stars cannot come from the disc. Were we to have carried out this test at other TMGS positions in the plane, the excess of

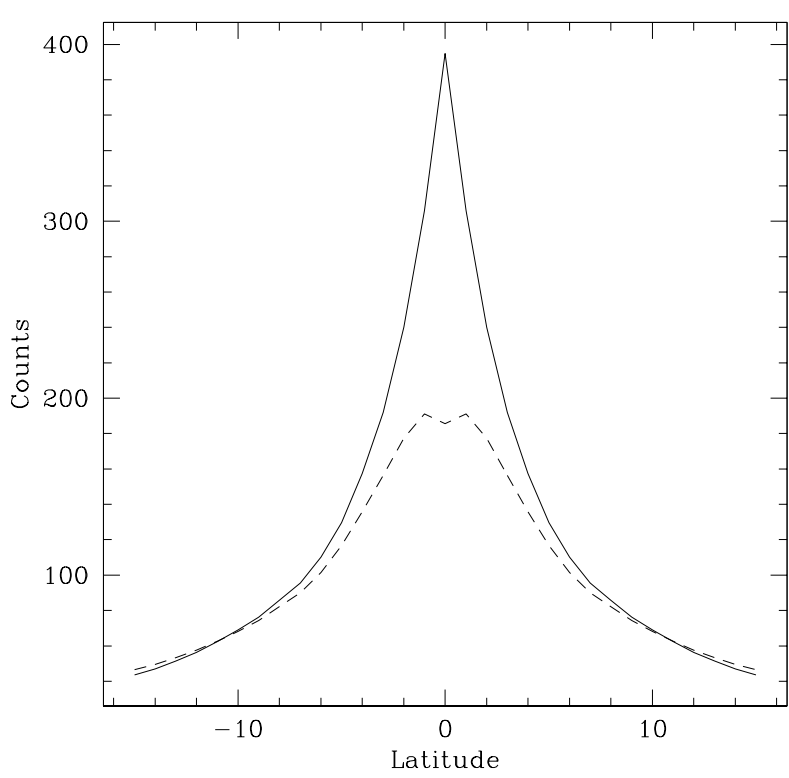

Figure 2. Two simplified (not SKY) model cuts at $l=65^{\circ}$ for $m_{K}=9$. The solid line shows an exponential disc (as in SKY) the dashed is based on $\operatorname{asch}^{2} z$. In both cases the SKY disc LF was used. The two curves have been normalized at $b=10^{\circ}$.

counts above the $\operatorname{sech}^{2} z$ law would be even larger than that shown in Figure 2.

We further note that the SKY model produces good fits to the DENIS $K$ star counts in the plane to $m_{K}=13.5$ (Ruphy et al. 1997), significantly deeper than the TMGS. In regions away from the Galactic bulge, the contribution from the disc will be at least an order of magnitude greater than from any other component, e.g. the arms. If the disc were not exponential, or at least very close to exponential, all the way to the plane, then SKY would significantly overestimate the counts on the plane.

The $\operatorname{sech}^{2} z$ function is strictly valid only for an isolated, self-gravitating, isothermal system. Self-consistent dynamical models of the disc (e.g. Haywood et al. 1997) show the dependence of the resulting z-distribution on star formation rate (SFR). For all but declining SFRs, the vertical distributions can mimic exponentials. It is our suspicion that the $\operatorname{sech}^{2} z$ function from a locally isothermal disc achieved its popularity in the context of overall light distributions across the planes of edge-on spirals at optical wavelengths (e.g. van der Kruit \& Searle 1982). In such a situation, heavy and patchy extinction militates against any meaningful comparison between a $\operatorname{sech}^{2} z$ law and observations and, far from the planes of such galaxies, one cannot distinguish between $\operatorname{sech}^{2} z$ and exponential functions. Perhaps the only redeeming feature of a $\operatorname{sech}^{2} z$ law is that it offers a null second spatial derivative at zero latitude, yet this is precisely the latitude range in which optical observations are invalid for the purposes of making such comparisons. Further, even in the field of edge-on external galaxies, there is evidence that the infrared light distributions follow exponential rather than $\operatorname{sech}^{2} z$ functions (e.g. Wainscoat et al 1989). 


\section{THE PLANE OF THE DISC}

For $|b|<2^{\circ}$, at all longitudes, the TMGS has more counts on the plane than are predicted by SKY. The profiles of counts (Figure B) show three distinct distributions at these latitudes:

- A distribution extending from about $|b|<4^{\circ}$ that is seen at all longitudes between $l=70^{\circ}$ and $-1^{\circ}$.

- A spiked distribution about $1^{\circ}$ wide centred on the plane at $l=21^{\circ}$ and $27^{\circ}$.

- The bulge at $l=-1^{\circ}$ and $l=7^{\circ}$ (which we treat in section 9).

\subsection{The Spiral Arms}

SKY predicts that the $2-\mu \mathrm{m}$ star counts should form a continuous distribution from $b=15^{\circ}$ to $b=1^{\circ}$ with the only serious deviations due to extinction in the plane. However, the scans $l=21^{\circ}, 31^{\circ}$ and $65^{\circ}$ all show a distinct transition at about $|b|=5^{\circ}$. (These can be seen both in the cuts [Figures B3, B5 and B8] and in the counts [Figures A3, A5, A7, D3, D5, D8 ].) The star counts for $|b|>5^{\circ}$ approximate an exponential very well but, at $b=5^{\circ}$, it is clear that a second contribution is required with a far smaller scale height than typifies the stars of the disc (i.e. an element rising more rapidly than the disc as latitude decreases). Figure 1(e) represents this phenomenon from the $l=65^{\circ}$ strip, some $3.5^{\circ}$ off the plane.

A feature so close to the plane has to be due to a young component. There are two possibilities to explain this distribution; a young disc or spiral arms. SKY already has a young component within its representation of the disc and these young stars $(\mathrm{O}, \mathrm{B}$ and $\mathrm{A})$ have a much smaller scale height than the older stars. Thus, if the extra sources in the plane were young disc stars, this would imply an enhancement in these populations. Indeed, examination of Figures B and $\mathrm{C}$ shows that, particularly at the brighter magnitudes, the numbers of young sources would need to be increased by orders of magnitude to reproduce the counts near the plane. This would create a major distortion of the LF, clearly recognizable in the local vicinity. Figure $\mathrm{C}$ shows the TMGS counts after subtracting the SKY disc. Note that, for both $l=31^{\circ}$ and $l=65^{\circ}$, the width of the non-disc source distribution is independent of magnitude, suggesting that these sources are grouped at a specific distance rather than spread along the line-of-sight. For the above reasons, we prefer to attribute the excess of stars on the plane to the arms, rather than to a boost in the young disc.

The prediction from SKY is that the arms are always substantially below the disc counts, although there are certainly locations in which the arms' contribution is second only to that of the disc. However, particularly at the brighter $m_{K}$ magnitudes $\left(m_{K}<7.5\right)$ the TMGS finds that the counts from the arms are at least as important as those from the disc within $2^{\circ}$ of the plane. The two major TMGS regions (i.e. with sufficient area to give reliable statistics at the brightest magnitudes) that run only through spiral arms and through none of the other inner Galaxy components are at $l=31^{\circ}$ and $l=65^{\circ}$. At $l=31^{\circ}, b=0^{\circ}$ at $m_{K}=7$, the TMGS has nearly three times SKY's predicted counts. At $l=65^{\circ}$ at $m_{K}=7$, the excess on the plane is only $50 \%$ but at $m_{K}=5$ the TMGS is again a factor of 3 too high. This can be clearly seen in Figure B.

By $m_{K}=9$ in both the $l=31^{\circ}$ and $65^{\circ}$ strips the arm-todisc ratio has dropped significantly. In the range $8<K<9$ at $b=0^{\circ}$ there are some 331 predicted disc sources compared with only $40 \mathrm{arm}$ sources. At $l=31^{\circ}$ the loss of contrast is not as great (540 disc sources to 350 arm sources) probably because this line of sight is almost tangential to the Scutum arm, looking into the inner Galaxy, whereas the $l=65^{\circ}$ line of sight roughly maintains a Galactocentric distance of $\mathrm{R}_{\odot}$ for over $8 \mathrm{kpc}$.

Another interesting conclusion can be reached by detailed comparison of the star counts at $l=31^{\circ}$ and $65^{\circ}$. In Figures C5 and C6, the dominant contribution to the discsubtracted counts is from the arms. At $m_{K}=5$, we see roughly the same absolute number (within a factor of 1.5) of luminous non-disc sources in both directions yet, by the fainter magnitudes (e.g. $m_{K}=9$ ), the $l=31^{\circ}$ line of sight shows about 6 times as many spiral arm sources as towards $l=65^{\circ}$. Thus, the LF of the spiral arms must be a function of Galactocentric distance, rather than constant as in SKY4. If this is truly the case, one might suppose that metallicity was implicated. There is a precedent for this argument in the observed confinement of the latest WC-type Wolf-Rayet stars to the immediate environs of the GC, a phenomenon attributed by Smith \& Maeder (1991) to the high metallicity near the Centre which biases evolution of massive stars to the formation of WC9 stars.

At $l=65^{\circ}$ (Figure C6), only the Perseus arm is crossed and at a Galactocentric distance of about $8.5 \mathrm{kpc}$. Here the excess is around 80 sources $\mathrm{deg}^{-2}$ to $m_{K}=8$. It is noticeable that the peak is not at $b=0^{\circ}$; rather the centre of the distribution is shifted some $1.5^{\circ}$ to positive latitude. Also, the observed counts drawn from the $l=37^{\circ}$ strip are parallel to, but above, SKY's predictions at all magnitudes. Both features are symptomatic of directions in which the spiral arms do not lie in the formal Galactic plane but arch above it. This same effect was detected in the IRAS counts by WCVWS, who were able to match it to specific regions in the H I sky (e.g. Weaver 1974) where neutral gas lies almost $1 \mathrm{kpc}$ off the plane. They cited, in particular, the $l=50^{\circ}$ to $90^{\circ}$ region, which includes the off-plane peak of TMGS $K$ counts along the $l=65^{\circ}$ strip.

Figure 3 shows the peak source count detected in each strip after the SKY disc has been subtracted. Vertical dashed lines are the points tangential to the Scutum and Sagittarius spiral arms. The aim is to show primarily the arm sources on the plane, therefore, at $l=7^{\circ}$ and $l=-1^{\circ}$ only sources to $m_{K}=6$ have been shown to avoid bulge sources. Also because of the spike (Section 6.2) the values at $l=21^{\circ}$ have been estimated as if the spike were not there and the region at $l=27^{\circ}$ has been ignored. The $l=31^{\circ}$ and $l=37^{\circ}$ lines of sight are very close together, yet the $l=31^{\circ}$ region has almost twice the number of arm sources as at $l=37^{\circ}$. The only significant difference is that the $l=31^{\circ}$ direction crosses the Sagittarius and Scutum arms whilst $l=37^{\circ}$ intercepts only the Sagittarius arm. The extra sources to $m_{K}=7$ and 8 suggest that just under half the excess sources can be attributed to the Scutum arm (i.e. about 350 sources $\mathrm{deg}^{-2}$ to $m_{K}=8$ on the plane). The effect of the arms on the counts can be clearly seen in all regions, in particular the influence of the number of arms which cut 


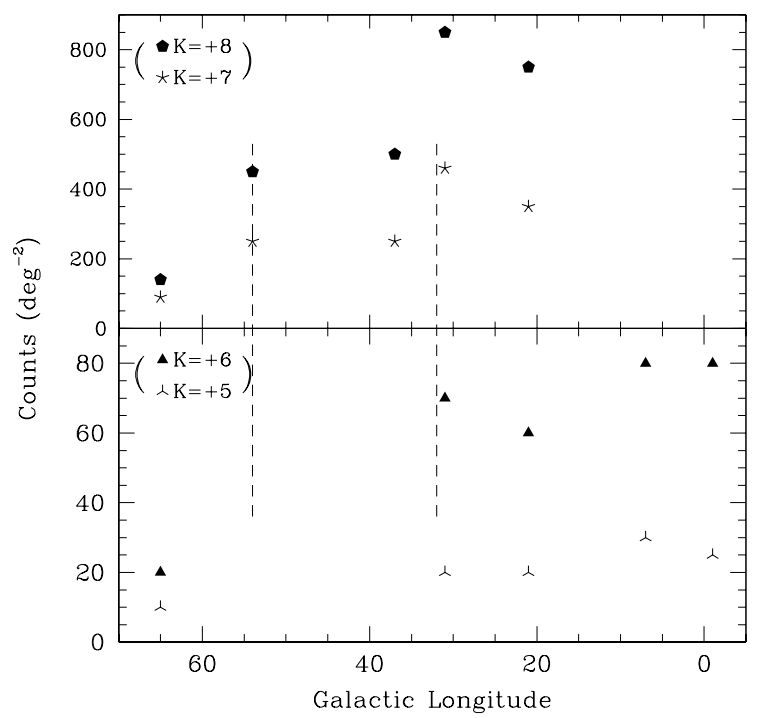

Figure 3. The peak cumulative count at each TMGS plane crossing after subtracting the SKY disc. The aim is to show those counts from the arms therefore the regions at giving a major contribution at fainter magnitudes. Also at $l=21^{\circ}$ the counts are the estimated counts if the "spike" (see below and Figure 3 of Hammersley et al. 1994) are ignored.

the line of sight. Directions crossing two arms have nearly twice the counts of those crossing only one. Furthermore, the inner spiral arms are far stronger than the arms at the distance of the solar circle (cf. the difference between $l=65^{\circ}$ and the other regions). Another curious feature of Figure 3 is that at $m_{K}=5$ and 6 the number of spiral arm sources does not alter significantly with longitude between $l=-1^{\circ}$ and $l=31^{\circ}$. Clearly there is a strong interplay between the distance to the arm and the LF but one would expect the $l=-1^{\circ}$ and $l=7^{\circ}$ cuts to be significantly less than the $l=31^{\circ}$ which is almost tangential to the Scutum arm.

We attribute these excess sources observed near the plane by the TMGS to the presence of an additional population in the arms, not present in SKY4. From the estimated distances to these spiral arms, this population would have to be of supergiant luminosity. Consequently, the spiral arms clearly provide an important contribution to the $K$ star counts, particularly towards the inner Galaxy.

\subsection{The "Spikes"}

The most dramatic deviations from the SKY model anywhere in the area covered here are the "spikes" on the plane at $l=21^{\circ}$ and $l=27^{\circ}$. Figures A3 and A4 portray the differential star counts at $l=21^{\circ}, b=0^{\circ}$, and $l=27^{\circ}, b=0^{\circ}$, where the excess is substantial at all magnitudes. If the SKY disc is removed (Figures $\mathrm{C} 3$ and $\mathrm{C} 4$ ), then the TMGS has 6 times the counts of the remaining SKY components at $l=21^{\circ}$. In the cuts (Figures B3 and B4), it can be seen that the distribution of the excess counts is very narrow, about $1^{\circ}$ wide, hence the term "spike". It is significant that the spike only appears at $l=21^{\circ}$ and $l=27^{\circ}$, there is no evidence for it at $l=31^{\circ}$ (or larger longitudes). Neither is there evidence for the spike at $l=7^{\circ}$ or $-1^{\circ}$ although the arms are clearly visible at the brighter magnitudes in both strips so the absence of the spike cannot be just a line of sight effect. This almost rules out the feature being due to a continuous feature circling the inner Galaxy. The implications of the spikes were discussed by Hammersley et al. (1994), who argued that these must be features associated with the inner $3-4 \mathrm{kpc}$ of the Galactic disc. The scale height must be around $40 \mathrm{pc}$, suggestive of a very young population, and the $M_{K}$ must attain at least -11 to -13 . Mikami et al. (1982) suggested that there is a clustering of supergiants at $l=27^{\circ}, b=0^{\circ}$. Garzón et al. (1997) obtained visible spectra of some of the brightest TMGS sources with optical counterparts, proving that there are a very high number of supergiants in this region. There are two possible explanations for what causes these spikes: bar or ring.

In order to match the IRAS 12 - and $25-\mu \mathrm{m}$ counts in this region WCVWS introduced another component, the molecular ring (Dame et al. 1987). However, it is clear that, even with this representation of the ring, SKY4 is well below the TMGS counts. The most significant deviations are confined to $|b| \leq 1.5^{\circ}$ on the $l=21^{\circ}$ strip, but persist to higher latitudes along the $l=27^{\circ}$ strip $\left(|b| \leq 3.5^{\circ}\right)$. Note that the slope of the observed counts in these regions more closely parallels that of SKY's ring component than the steeply sloped disc counts. Were we to posit that an additional stellar population attends the ring, close to the plane, then this population must contribute some 5 times SKY's predicted ring counts near $l=21^{\circ}$ but almost an order of magnitude more than SKY's ring near $l=27^{\circ}$. The excess of sources above SKY's expectation is very similar on the $l=27^{\circ}$ and $21^{\circ}$ strips, but shows a conspicuous additional bias toward very bright sources near $l=27^{\circ}$.

We note that Ruphy et al. (1997) describe an analysis of DENIS near-infrared counts using a new version of SKY, with a non-uniform elliptical ring that replaces the old circular molecular ring in SKY4. In a later paper we plan to exercise SKY5 (after implementing all the changes discussed in the present paper) to investigate what TMGS counts tell us about the geometry of this ring. In brief, DENIS counts probe the lower-density portions of this ring and help to constrain the overall geometry of the ellipse. But TMGS offers direct lines of sight across the first quadrant dense ansa included in SKY5. Given sufficient TMGS strips, and new deeper $J H K$ images, the density enhancements around this new ring should be definable. The inclusion of these star-rich ansae provides SKY with a measure of asymmetry (triaxiality) not present in earlier versions, and can be used to provide a more formal examination of the bar. However, we defer this discussion to a future paper using new data and further developments of SKY.

Hammersley et al. (1994) suggested that such spikes could be associated with a possible bar distribution on the grounds that, where the bar interacts with the disc, there is a high probability of strong star formation. Note this "bar" is not the triaxial bulge, often called a bar, but rather is a sticklike bar with a half-length of nearly $4 \mathrm{kpc}$ and an angle of $75^{\circ}$ to the GC-sun line. Neither can the star formation be related to the triaxial bulge somehow triggering star formation in the inner disc (as suggested by Freudenreich 1998) as the geometrical constraints alone make this nearly impossible. Hammersley it et al. (1994) also examined the COBE 2.2- 
$\mu \mathrm{m}$ surface brightness maps and argued that such a bar far more simply and naturally predicts the observed features (both images and star counts), whilst the form of a ring would have to be contrived using ad hoc solutions to make it fit.

It is beyond the scope of the current paper to engage in a discussion of ring versus bar. To make any significant advance, new data are required. However, it should be noted that many external bars have strong ansae of star formation at either ends and so the discussions in the previous two paragraphs are not mutually exclusive.

\section{THE BULGE}

Two TMGS strips run through the bulge: those at $l=-1^{\circ}$ and $l=7^{\circ}$. However, when examining these two regions, one must remember that the strips cut the plane at an appreciable angle so there is a significant variation in $l$ as well as $b$, which is important this close to the GC. These are the most complex regions to analyse because disc, arms, and bulge contribute to the counts. The first step is to subtract the SKY disc to highlight the bulge population. This is entirely reasonable because SKY gives a good fit to the exponential disc, and because of the high bulge-to-disc ratio fainter than $m_{K}=7$ (e.g. near the plane, there are twice as many bulge stars as disc stars).

Figures B1 and B2 show the cuts for the two strips; Figures $\mathrm{C} 1$ and $\mathrm{C} 2$ show the residuals after subtracting SKY's disc from the TMGS counts. At $m_{K}=5$ and 6 the residuals in the TMGS are clearly due to spiral arm sources because they are as narrow in spatial extent, and as large in size, as the residuals at $l=31^{\circ}$. However, at $m_{K}=6$, SKY's predicted residuals clearly show a wider distribution than seen in the TMGS, due to the predicted bulge sources. Even at $l=7^{\circ}$ the SKY residual is far wider than seen in the TMGS, yet by $m_{K}=9$ the residuals are far closer together. The explanation for these discrepancies is that SKY's bulge LF has too many of the most luminous sources. A quantitative analysis of the $K \mathrm{LF}$, based on the TMGS, is presented in LópezCorredoira et al. $(1997,1999)$ and shows that SKY's bulge LF starts 1 to 2 magnitudes too bright at $K$. Consequently, it is planned to implement a new LF for the bulge and to re-examine this issue in the future.

The SKY bulge is axially symmetric, with a projected axial ratio of 0.6. Therefore, if one were to draw ellipses of constant predicted bulge star counts, then these would have a ratio of major-to-minor axis of 0.6 ; i.e. all positions on the locus $\sqrt{0.6^{2} l^{2}+b^{2}}$ would have the same star counts. The bulge is the only component which has this type of projected spatial distribution and so plotting this function is an unambiguous test of whether the TMGS is actually detecting bulge sources. It should be noted that as the function $\sqrt{0.6^{2} l^{2}+b^{2}}$ is always positive this means that the line for a TMGS strip will double back on itself as it crosses the plane. Figure 4 shows SKY's bulge counts plotted against $\sqrt{r a t^{2} l^{2}+b^{2}}$ (right column) together with the TMGS strips (left column) through $l=7^{\circ}$ (dashed line) and $l=-1^{\circ}$ (solid line) for various ratios, rat, between 1.5 and 0.2 . In both cases this is after subtracting the SKY disc and for $m_{K}=9$ so that the bulge will dominate all the remaining components. More than a few degrees from the GC, the best fit for the bulge (i.e. the lines at the same position most nearly overlie each other is for a projected axial ratio of 0.6). There is some evidence from the central few degrees (Figure $4, l=-1^{\circ}$ ) that the fit is better for an axial ratio of 1.0, but this is consistent with a triaxial bulge (as will be discussed below), which would depress the portion from negative longitudes.

Figure 5 shows the same type of plot as Figure 4, this time varying the $K$-magnitude at a fixed ratio of 0.6 . The left column shows the TMGS whilst the right column shows the ratio of (TMGS-SKY disc) to (SKY total-SKY disc). At $m_{K}=5$, where there is no bulge component, there are two separate peaks caused by the spiral arm component in the plane. Spiral arms clearly will not follow the bulge locus. At $m_{K}=6$, SKY (as discussed above), predicts that there should be a bulge-like component but the TMGS plots show that the only significant population is that of the arms. For this reason, apart from the plane crossings, the TMGS is below the counts predicted by the model. $m_{K}=7$ is a transitional magnitude where the bulge begins to become important, although in the plane there is still a significant arm component. On average, the TMGS residuals are below the predicted SKY residuals; i.e. the TMGS bulge counts are below those of SKY. On average at $m_{K}=8$ and 9 away from the plane, the ratio of SKY to TMGS is about 1 suggesting that by these magnitudes the SKY LF is close to that observed by the TMGS. Note that the peak in the ratio where the TMGS strips cut the plane (where the curves for each strip double back on themselves) at $m_{K}=8$ and 9 is caused by the model overestimating the extinction in the inner Galaxy (see Section 4.2).

SKY predicts that the bulge distribution in the two cuts should be asymmetric due to the change in $l$ as well as $b$. However, a comparison of the TMGS residuals, taking account of the discrepancy in the LF, shows that for $b<0^{\circ}$ $\left(l>0^{\circ}\right.$ by comparison with the point at $\left.b=0^{\circ}\right)$ the TMGS is invariably above SKY, whereas for $b>0^{\circ}\left(l<0^{\circ}\right)$ the TMGS is well below SKY (Figures B1, B2, C1 and C2). It should be noted that the effect is very obvious particularly at $m_{K}=7$ and 8 and so we are not discussing some subtle feature in the counts. Initially it was supposed that this was due to the extinction by major dark cloud systems above the Galactic plane in this direction. However, recent studies have shown that in general there is insufficient extinction in these clouds to account for the observed effect we see (Mahoney 1998). Typically, the additional $A_{V}$ above the plane, as opposed to below it, is $1 \mathrm{mag}$ (i.e. an extra $A_{K}$ of about $0.1 \mathrm{mag})$. The asymmetry seen in the TMGS would require a difference of extinction in $K$ of at least $0.5 \mathrm{mag}$, which is impossible to attribute to extinction alone. Figure 5 shows that at $l=-1^{\circ}$ (the solid line) the difference between the TMGS/SKY ratio for $b<0^{\circ}\left(l>0^{\circ}\right.$, the upper part of the solid line) and $b>0^{\circ}\left(l<0^{\circ}\right)$ increases with distance from the Galactic plane (between 2 and 7 on the $\mathrm{x}$-axis). However, the total extinction, as well as the difference in extinction above and below the plane, must decrease away from $b=0^{\circ}$. Hence, if the effect were due to extinction both the upper and lower parts of the solid line would come together with increasing distance from $b=0^{\circ}$, and not diverge as is seen. Furthermore, if the differential extinction above and below the Galactic plane were significant, it would clearly show in 

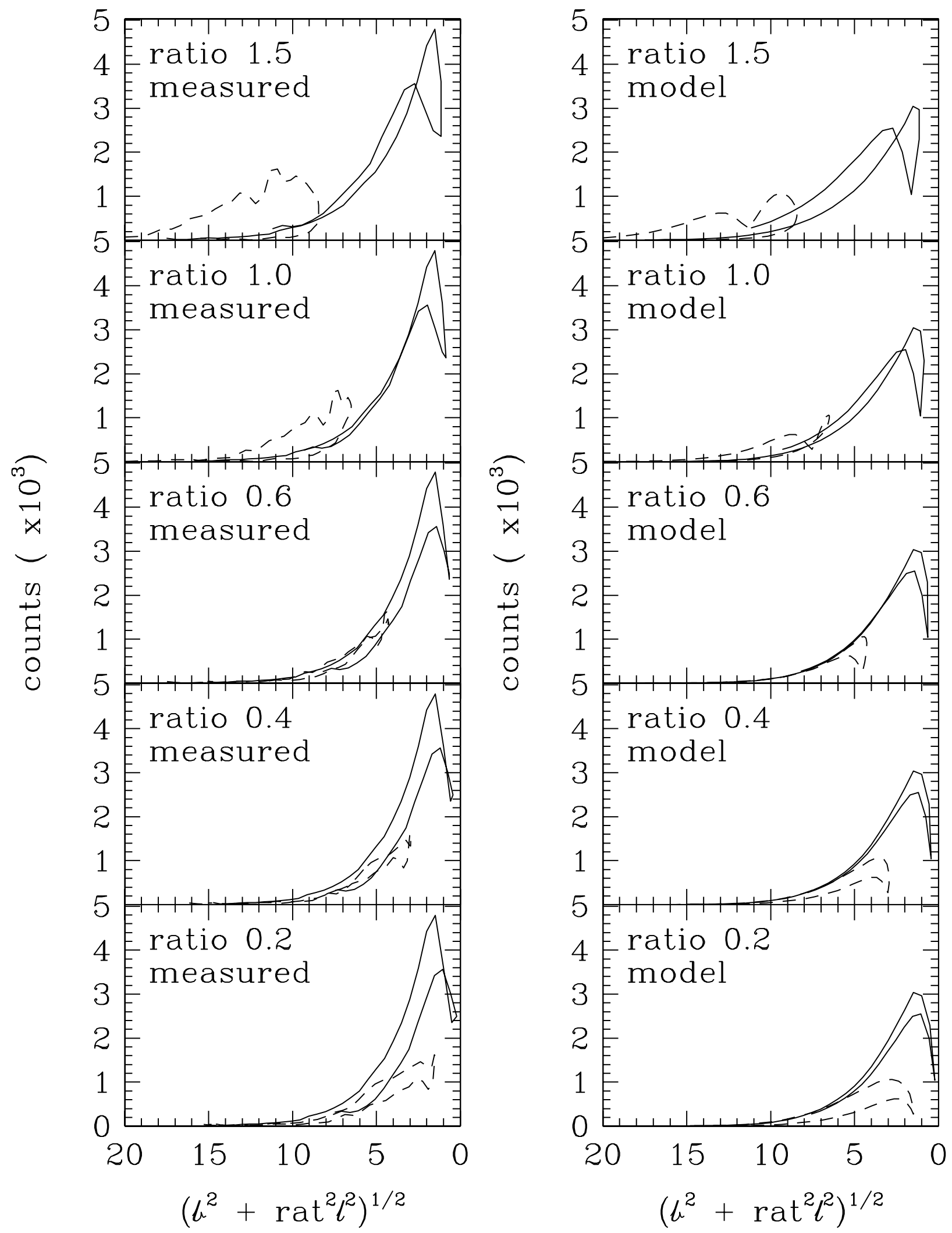

Figure 4. The left column shows the TMGS counts after subtracting the SKY disc plotted against $\left(r a t^{2} l^{2}+b^{2}\right)^{0.5}$ for various ratios (rat). The right column shows SKY total - SKY disc plotted in the same manner. The strips are for $l=-1^{\circ}$ (solid line) and $l=7^{\circ}($ dashed line). 

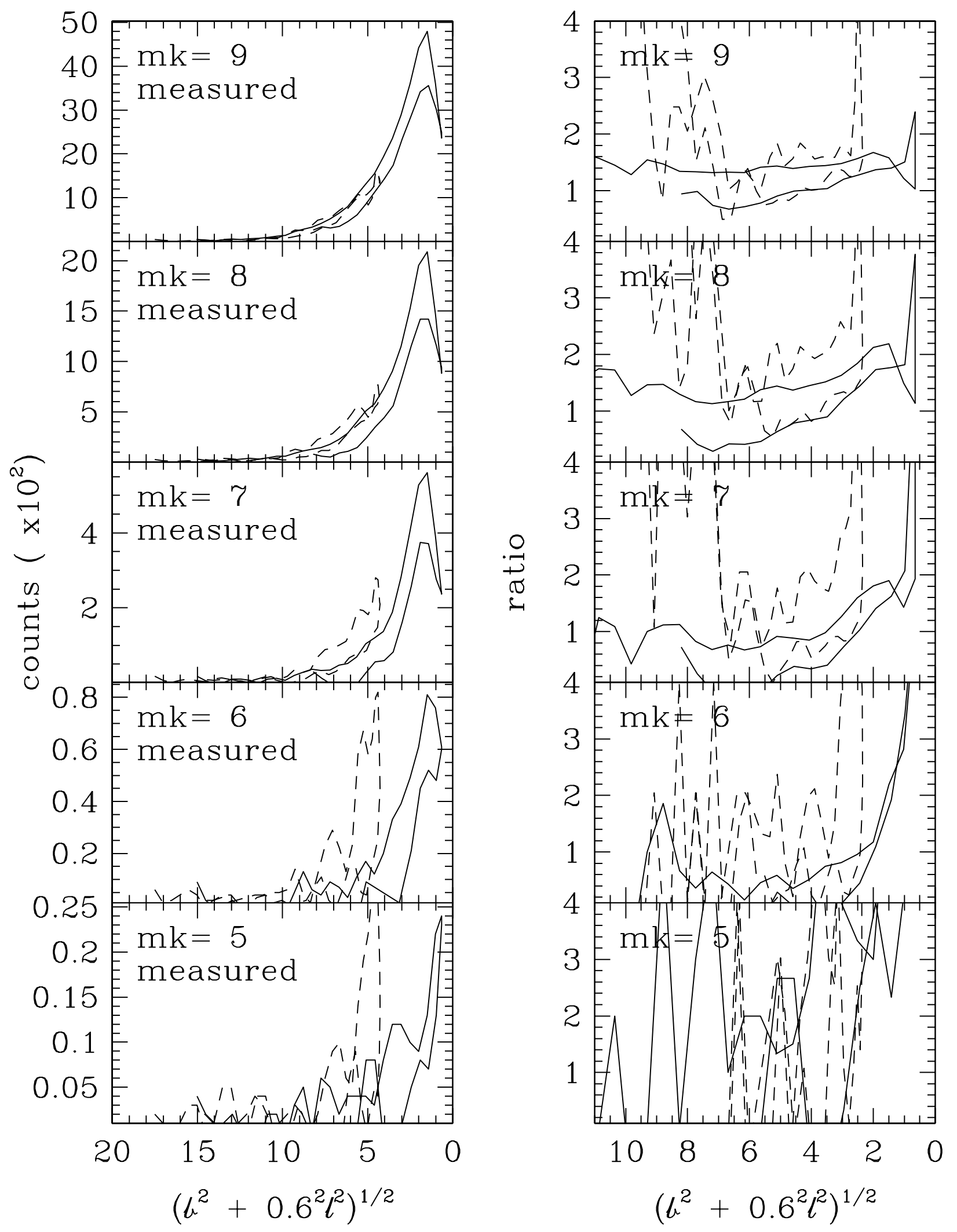

Figure 5. The left column shows the TMGS counts after subtracting the SKY disc plotted against $\left(0.6^{2} l^{2}+b^{2}\right)^{0.5}$ for various ratios $K$ magnitudes. The right column shows the ratio of (TMGS - SKY disc) to (SKY total - SKY disc ) also plotted against $\left(0.6^{2} l^{2}+b^{2}\right)^{0.5}$. The strips are for $l=-1^{\circ}$ (solid line) and $l=7^{\circ}$ (dashed line). 
the $C O B E 2.2-\mu \mathrm{m}$ surface brightness maps, which is not the case (see Freudenreich 1998).

A closer analysis of the difference between SKY and the TMGS shows that, at $m_{K}=8$ and 9 (where the LFs are in closer agreement), the TMGS is above SKY for $l>0^{\circ}$ and below SKY for $l<0^{\circ}$. Furthermore, at $m_{K}=7$, where the asymmetry in the TMGS is the greatest, the distribution is more bulge-like for $b<0^{\circ}$ (i.e. greater $l$ ) than $b>0^{\circ}$, where the distribution is almost narrow enough to be due solely to spiral arms, particularly in the $l=-1^{\circ}$ strip. A number of papers have argued that the bulge is triaxial with the near end in the first quadrant at an angle of $12^{\circ}$ to $30^{\circ}$ to the GC-Sun line of sight (Binney et al. 1991: Dwek et al. 1995: Freudenreich 1997: López-Corredoira et al. 1997). This would on average make the bulge sources at $l>0^{\circ}$ closer than at $l<0^{\circ}$. If the bulge were triaxial, we would expect asymmetries in the TMGS star counts. The sources from the closer part of the bulge $\left(l>0^{\circ}\right)$ would appear at a significantly brighter magnitude than those with $l<0^{\circ}$. A rough estimate suggests a magnitude difference as high as $0.5 \mathrm{mag}$. The steep rise in the bulge LF for the brightest sources means the counts cut on very sharply around $m_{K}=6.5$, but then rise less steeply from $m_{K}>7.5$. Thus we would expect to see a very asymmetric distribution in the $m_{K}=7$ cut but a far more symmetric one at $m_{K}=9$, precisely as seen in the TMGS counts. Similarly, the ratios shown in Figure 5 have the features that one would expect when ratioing star counts from a triaxial bulge with those from an axisymmetric model; i.e. the more positive the longitude, the more the counts rise above the predicted values and the more negative the longitude, the more the counts fall below the predictions. $K$ star counts give access to the LF so we suggest that an analysis of the bulge based on star counts is likely to be far more sensitive than one based solely on surface brightness maps because it avoids the ambiguities in the size and orientation of the triaxial bulge inherent in an analysis of its surface brightness (Zhao 1997). Recently LópezCorredoira et al. $(1997,1999)$ have analysed the TMGS star counts by inverting the fundamental star count equation very different from the qualitative analysis presented here and have found that the bulge is triaxial with an angle to the GC-Sun line of $12^{\circ}$.

For the innermost $\operatorname{strip}\left(l=-1^{\circ}\right)$ almost sampling the GC, the latitude range in which there is statistically significant deviation from SKY that cannot arise from off-plane dark clouds, is confined to $|b| \leq 2.0^{\circ}$ (Figures A1, B1 and D1). From these plots, it is apparent that, if the bulge component were simply enhanced by factor of 2 , the total predicted curve would much more satisfactorily match the counts. This bears directly on the bulge LF implicit within SKY. One can see from Figure $1(\mathrm{~g})$ how it is possible, quite directly, to probe this LF because the observed counts are well-matched by SKY except in the restricted range $8.4>m_{K}>6.2$. TMGS counts exceed SKY's here by about $50 \%$ at most, but this is naturally attributable to the shape of the bulge LF which declines abruptly at bright $K$ magnitudes. We note, however, that there is some interplay between changes in the density function and changes in the bulge LF.

\section{CONCLUSIONS}

We conclude that, overall, SKY provides a very reliable simulator of the TMGS Galaxy. Only rarely are the predictions wrong by as much as $50 \%$ and, in all these cases, there is a fairly obvious change that can be implemented in SKY to match the observations by more realistically representing the astrophysical situation. Consequently, the wide-area coverage offered by TMGS provides substantive constraints on the model. This potential interaction between SKY and the real Galaxy is extremely valuable in the development of a better model and in better understanding the structures in the inner Galaxy and their relative importance to $K$ star counts.

Specific changes that we plan to investigate in more detail and to implement in a new version of SKY are: the inclusion of an additional population of supergiants in the cores of the spiral arms; a modified LF for the bulge; a triaxial bulge; more non-coplanar arms and plane than represented by WCVWS; and the non-uniformly dense elliptical ring.

Perhaps the single most important result is that the star counts at $2.2 \mu \mathrm{m}$ can clearly be modelled using simple analytical functions to describe the 4 Galactic components of disc, arms, bulge and ring.

\section{ACKNOWLEDGMENTS}

The Carlos Sánchez Telescope is operated on the island of Tenerife by the Instituto de Astrofísica de Canarias in the Spanish Observatorio del Teide of the Instituto de Astrofísica de Canarias.

\section{REFERENCES}

Bahcall J. N., 1986, ARA\&A, 24, 577

Beichman C. A., 1987, ARA\&A, 25, 521

Binney J., Gerhard O., Stark A., Bally J., Uchida K., 1991, MNRAS, 252, 210

Calbet X, Mahoney T., Garzón F., Hammersley P. L., 1995, MNRAS, 276, 301

Clemens D. P., Sanders D. B., Scoville N. Z., 1988, ApJ, 327, 139

Cohen M., Sasseen T. P., Bowyer S., 1994 ApJ 427848

Cohen M., 1994, AJ, 107, 582

Cohen M., 1995, ApJ, 444, 874

Combes F., 1991, ARA\&A, 191, 195

Dame, T. M. et al. 1987, ApJ, 327, 706

De Grijs R., Peletier R. F., van de Kruit P. C., 1997, A\&A, 327, 966

Dwek et al 1995, ApJ, 445, 716

Eaton N., Adams D. J., Giles A. B., 1984, MNRAS, 208, 241

Epchtein N., 1998, in N. Epchtein ed, The impact of near-infrared sky surveys on galactic and extragalactic astronomy, Kluwer Academic Publishers, Dordrecht. p. 3

Freeman K.C., 1987, ARA\&A, 25, 603

Freudenreich H. T., 1998, ApJ, 492, 495

Garzón F., Hammersley P. L., Mahoney T., Calbet X., Selby M. J., Hepburn I., 1993, MNRAS, 264, 773

Garzón F., López-Corredoira M., Hammersley P. L., Mahoney T., Calbet X., Beckman J. E., 1997, ApJ, 491L, 31.

Gilmore G., Wyse R. F. G., Kuijken K., 1989, ARA\&A, 27 ,555

Hammersley P. L., Garzón F., Mahoney T., Calbet X., 1994, MNRAS, 269, 753

Hammersley P. L., Garzón F., Mahoney T., Calbet X., 1995, MNRAS, 273, 206 
Hammersley P. L., Garzón F., Mahoney T., Calbet X, LópezCorredoira M., 1996 in D.L.Block and J.M.Greenberg ed., New Extragalactic Perspectives in the New South Africa, Kluwer Academic Publishers, Dordrecht, p. 534

Hammersley P. L., Garzón F., Mahoney T., López-Corredoira M., 1998, in N. Epchtein ed, The impact of near-infrared sky surveys on galactic and extragalactic astronomy, Kluwer Academic Publishers, Dordrecht, p. 63

Haywood, M., Robin, A. C., Creze, M. 1997, AA, 320, 428

Kawara K., Kozasa T., Sato S., Kobayashi, Y., Okuda H., Jugaku J., 1982, PASJ, 34, 389

López-Corredoira M., Garzón F., Hammersley P. L., Mahoney T., Calbet X., 1997, MNRAS, 292, 15L

López-Corredoira M., Garzón F., Hammersley P. L., Mahoney T., Calbet X., 1999, MNRAS, submitted.

Mahoney, T., 1998, in preparation

Majewski S. R., 1993, ARA\&A, 31, 575

Mikami T., Ishida K., Hamajima K., Kawara K., 1982, PASJ, 42 223

Neugebauer G., Leighton R. B., 1969, Two Micron Sky Survey. NASA SP-3047, GPO, Washington DC

Paul E. R., 1993, The Milky Way Galaxy and Statistical Cosmology, 1890-1924. Cambridge University Press, Cambridge.

Reid N., 1993, in S. R. Majewski, ed, Galaxy Evolution: The Milky Way Perspective. San Francisco, ASP, p. 37

Robin A. C., Crézé M., Mohan V. 1992, A\&A, 265, 32

Ruphy, S. et al. 1997, A\&A, 326, 597

Schmidt-Kaler T., 1977, Vistas Astron., 19, 69

Smith, L. F. \& Maeder, A. 1991, A\&A, 241, 77

Skrutskie M., 1998, in N. Epchtein ed, The impact of near-infrared sky surveys on galactic and extragalactic astronomy, Kluwer Academic Phblishers, Dordrecht. p. 11

Wainscoat R. J., Cohen M., Volk K., Walker H. J., Schwartz D. E., 1992, ApJS, 83, 111 [WCVWS]

Wainscoat R. J., Freeman K. C., Hyland A. R., 1989, ApJ, 337, 163

Walker H. J., Cohen M.,Volk, K., Wainscoat R. J., Schwartz D. E., 1989, AJ, 98, 2163

Weaver H. 1974, in Highlights of Astronomy, ed G. Contopoulos, IAU,p423

van der Kruit P. C., Searle L., 1982, A\&A, 110, 79

van de Kruit P. C., 1988, A\&A, 192, 117

Zhao, H. S, 1997 astrphy9705046

\section{APPENDIX A: THE PLOTS SHOWING THE COMPARISON BETWEEN TMGS AND SKY}

The appendix presents the majority of the the plots showing the detailed comparison between TMGS and SKY. From these plots it is possible to see how the relative importance of the various Galactic components vary with magnitude and position.

Figures A1 to A7 (9 plots) illustrate our interpretations of the counts by direct comparisons with the predictions of SKY. The plots are for a positions every few degrees along each scan (the full data is presented in figure D). Differential counts were scaled per square degree per magnitude, using entirely independent TMGS data in 0.2-mag wide bins. The counts arising from the separate geometric components are distinguished as follows: solid line - total count; dotted or less heavy line - disc; long dashes - spiral arms, local spurs and Gould's Belt; short dashes - bulge; short dashes and dots - molecular ring; long dashes and dots - halo. The position for each area is given in each plot. See section 3 and Tables 2 and 3 for more details.
Figures B1 to B9 show in the left column the cumulative star counts from the TMGS for each magnitude between $m_{K}=5$ and 9 plotted as the solid line. The longitude at which the strip crosses the plane is given in the upper box. The long dashed line shows the total prediction from SKY and the dotted line the counts from the SKY disc alone. In the right column is shown the ratio of TMGS counts to SKY total counts for each magnitude.

Figures $\mathrm{C} 1$ to $\mathrm{C} 6$ show the cumulative counts after subtracting the SKY disc from each magnitude between $m_{K}=5$ and 9. The longitude at which the strip crosses the plane is given in the upper box. The solid line is TMGS - SKY disc, The long dashed line is SKY total - SKY disc and the dotted line is the SKY bulge.

Figures D1 to D9 (33 plots) illustrate our interpretations of the counts by direct comparisons with the predictions of SKY every half a degree in latiude along the full TMGS scans. Differential counts were scaled per square degree per magnitude, using entirely independent TMGS data in 0.2-mag wide bins. The counts arising from the separate geometric components are distinguished as follows: solid line - total count; dotted or less heavy line - disc; long dashes - spiral arms, local spurs and Gould's Belt; short dashes bulge; short dashes and dots - molecular ring; long dashes and dots - halo. The position for each area is given in each plot. See section 3 and Tables 2 and 3 for more details. Due to the number of plots these are only available electronically. 

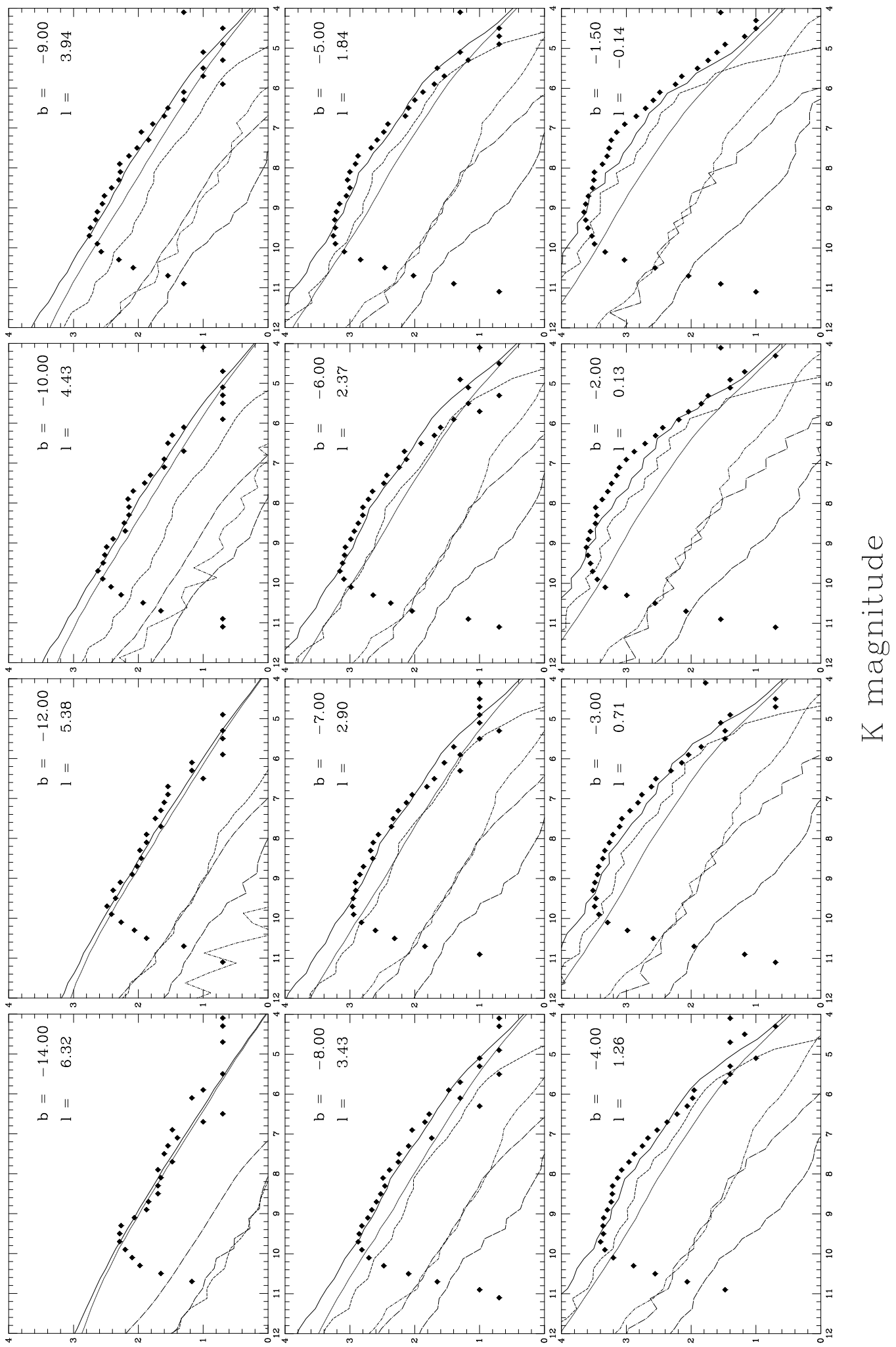

$\left(z-8 \partial p_{\text {I- }}\right.$ (8еu $)$ N 801

Figure A1a: TMGS differential star counts (diamonds) plotted on top of the SKY predictions for the strip crossing the plane at $l=-1^{\circ}$. 

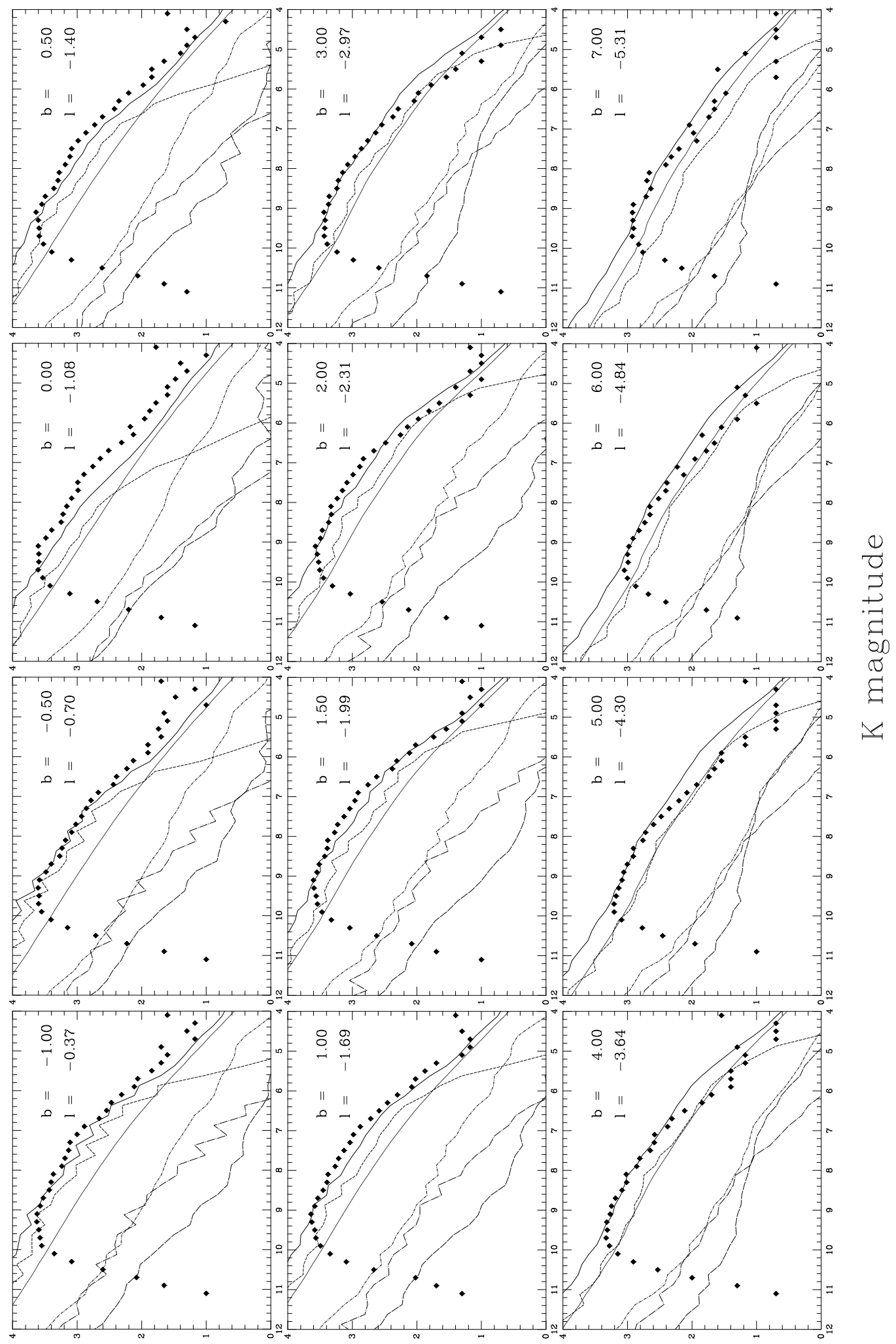

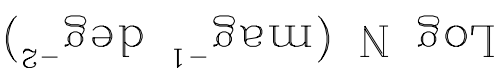

Figure A1b: TMGS differential star counts (diamonds) plotted on top of the SKY predictions for the strip crossing the plane at $l=-1^{\circ}$. 

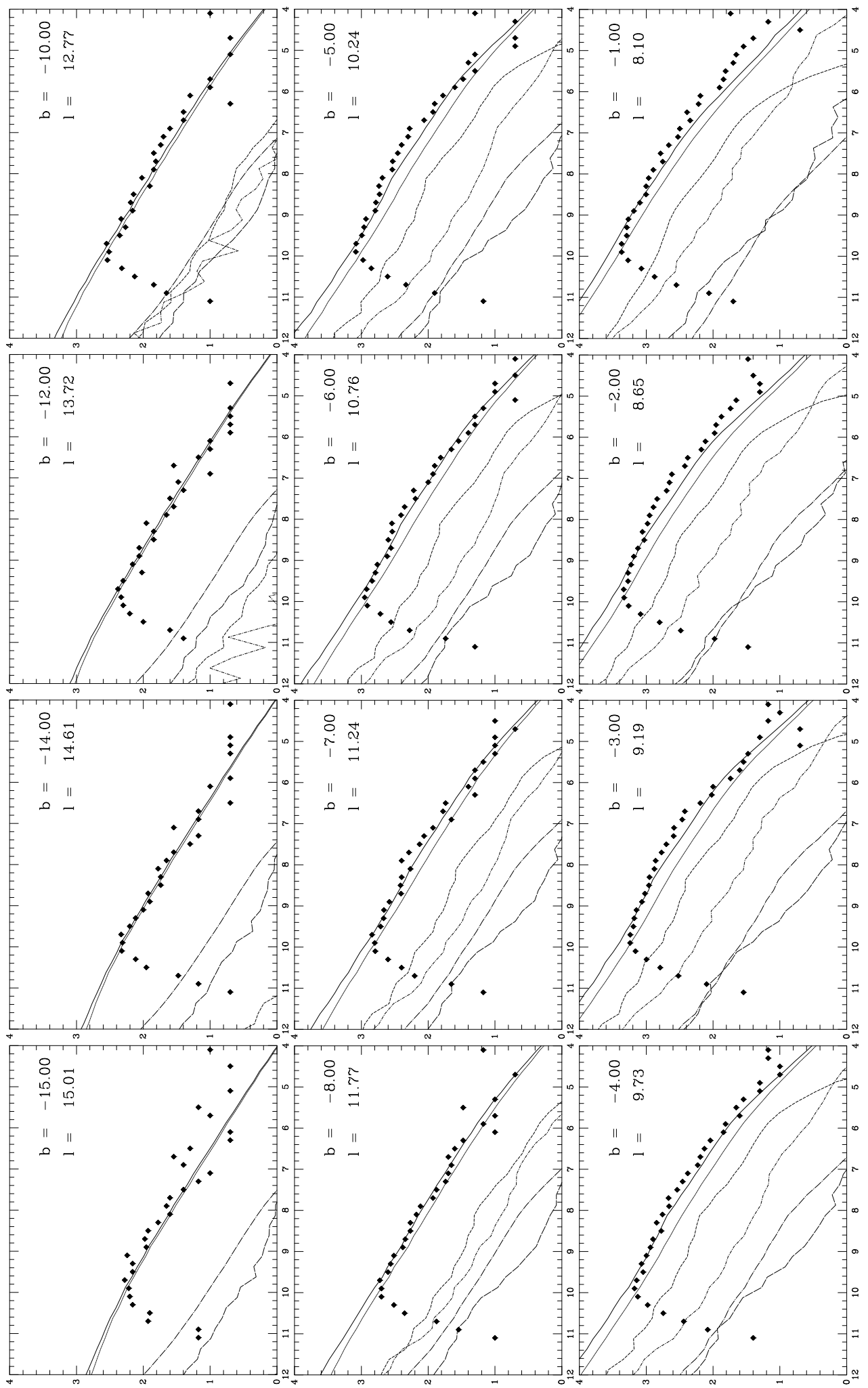

$\left(z-8 \partial p_{\text {I- }}\right.$ (8еu $)$ N 801

Figure A2a: TMGS differential star counts (diamonds) plotted on top of the SKY predictions for the strip crossing the plane at $l=7^{\circ}$. 


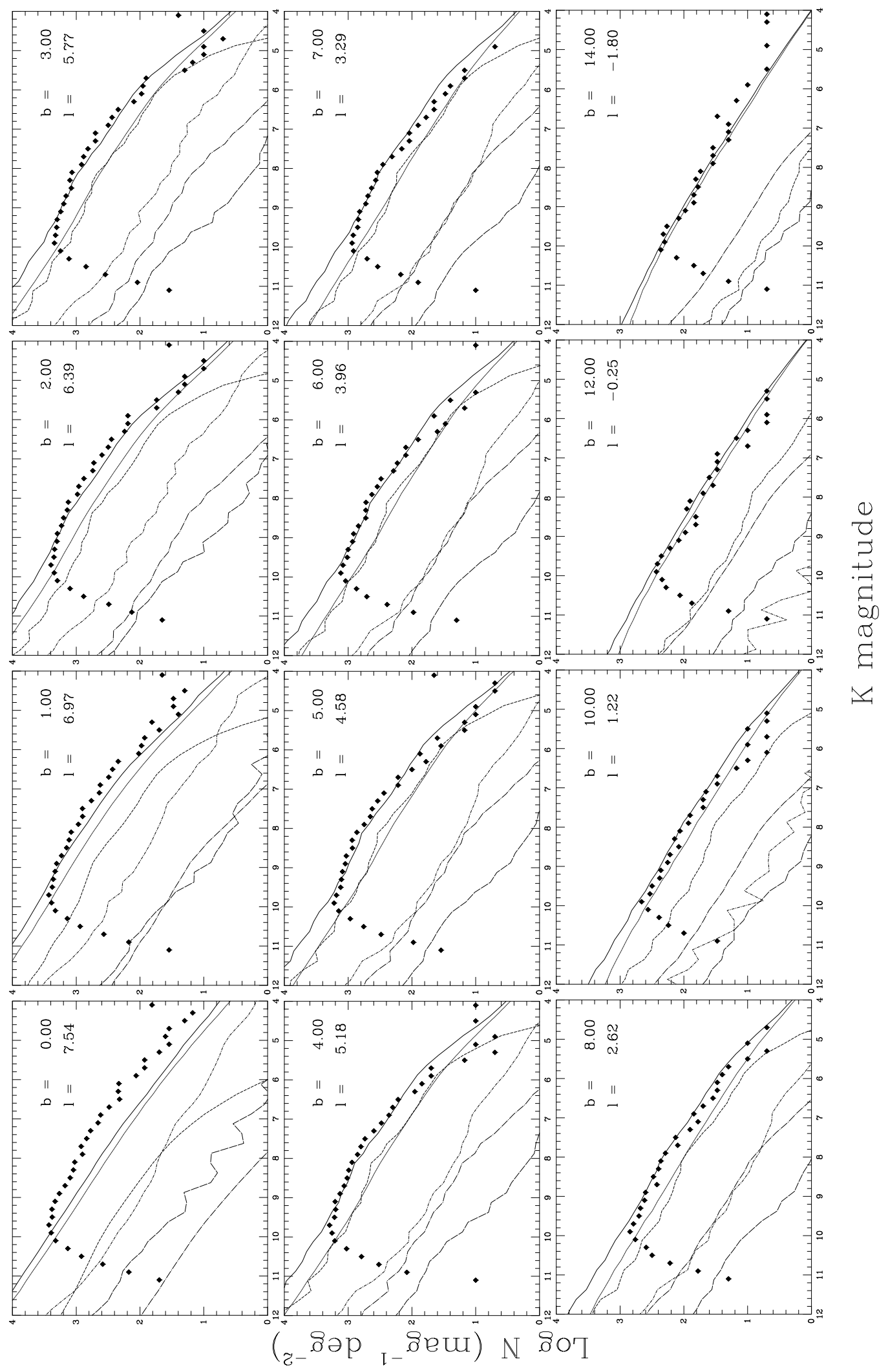

Figure A2b: TMGS differential star counts (diamonds) plotted on top of the SKY predictions for the strip crossing the plane at $l=7^{\circ}$. 

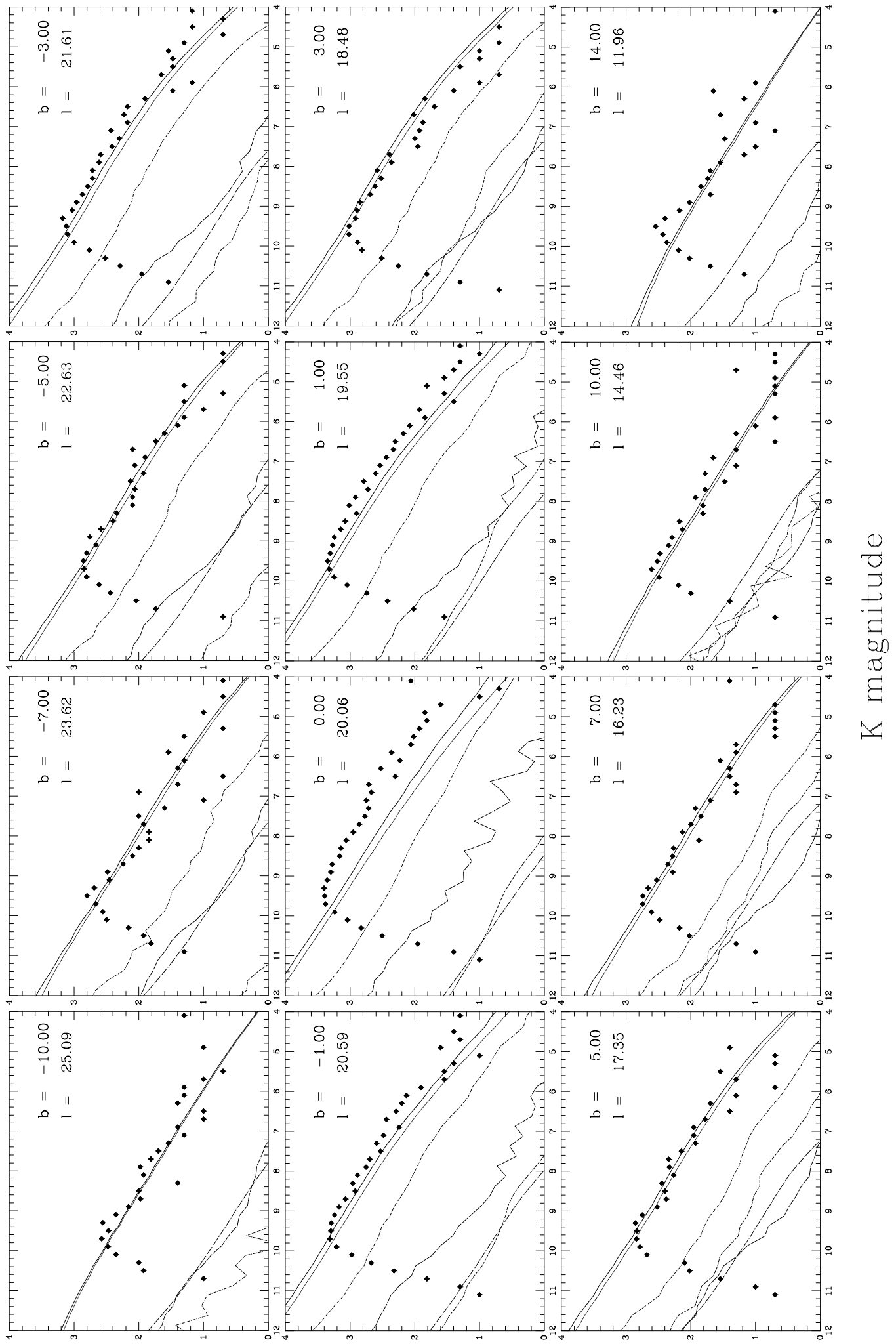

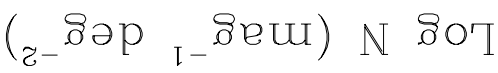

Figure A3: TMGS differential star counts (diamonds) plotted on top of the SKY predictions for the strip crossing the plane at $l=21^{\circ}$. 

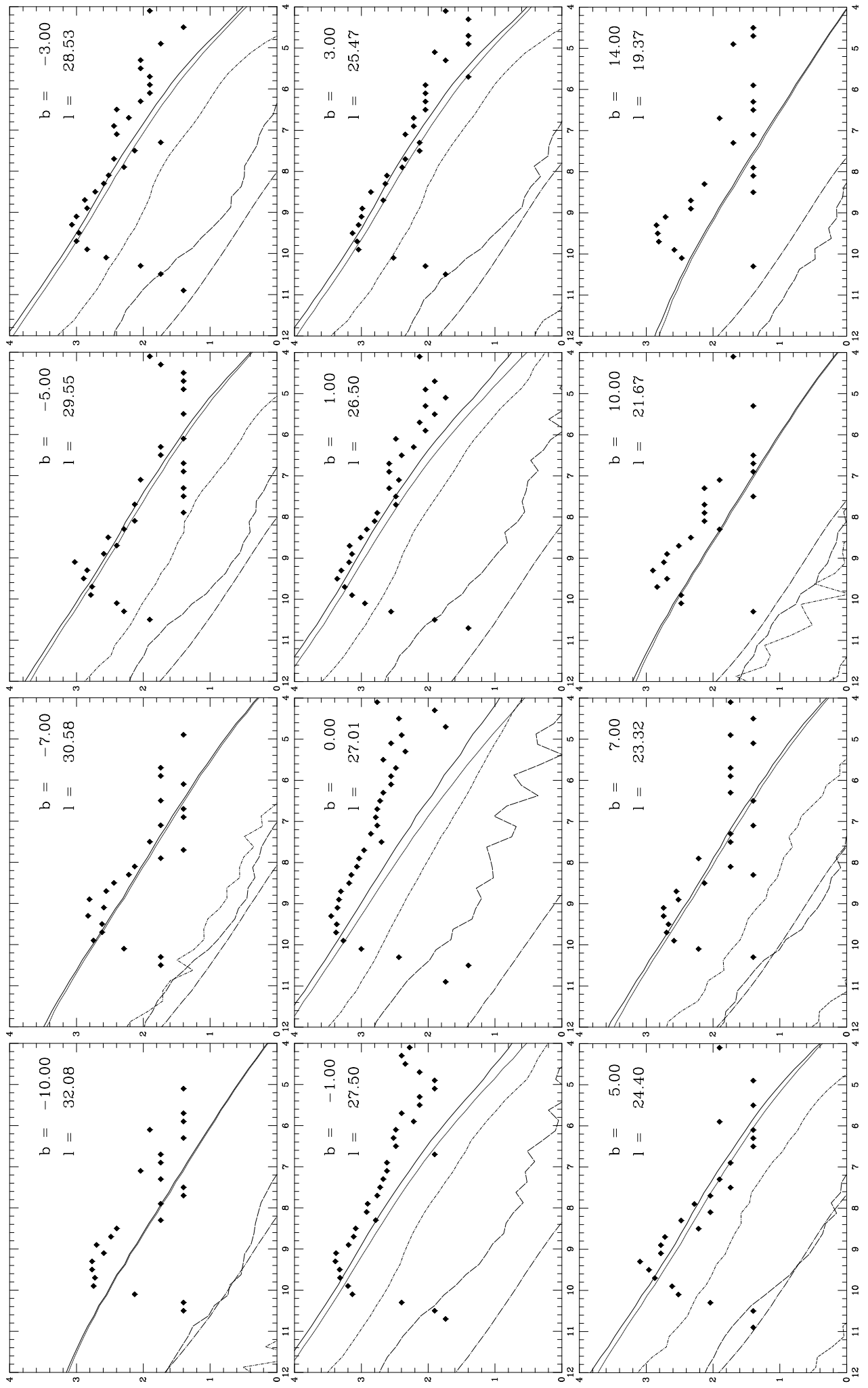

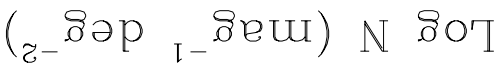

Figure A4: TMGS differential star counts (diamonds) plotted on top of the SKY predictions for the strip crossing the plane at $l=27^{\circ}$. 

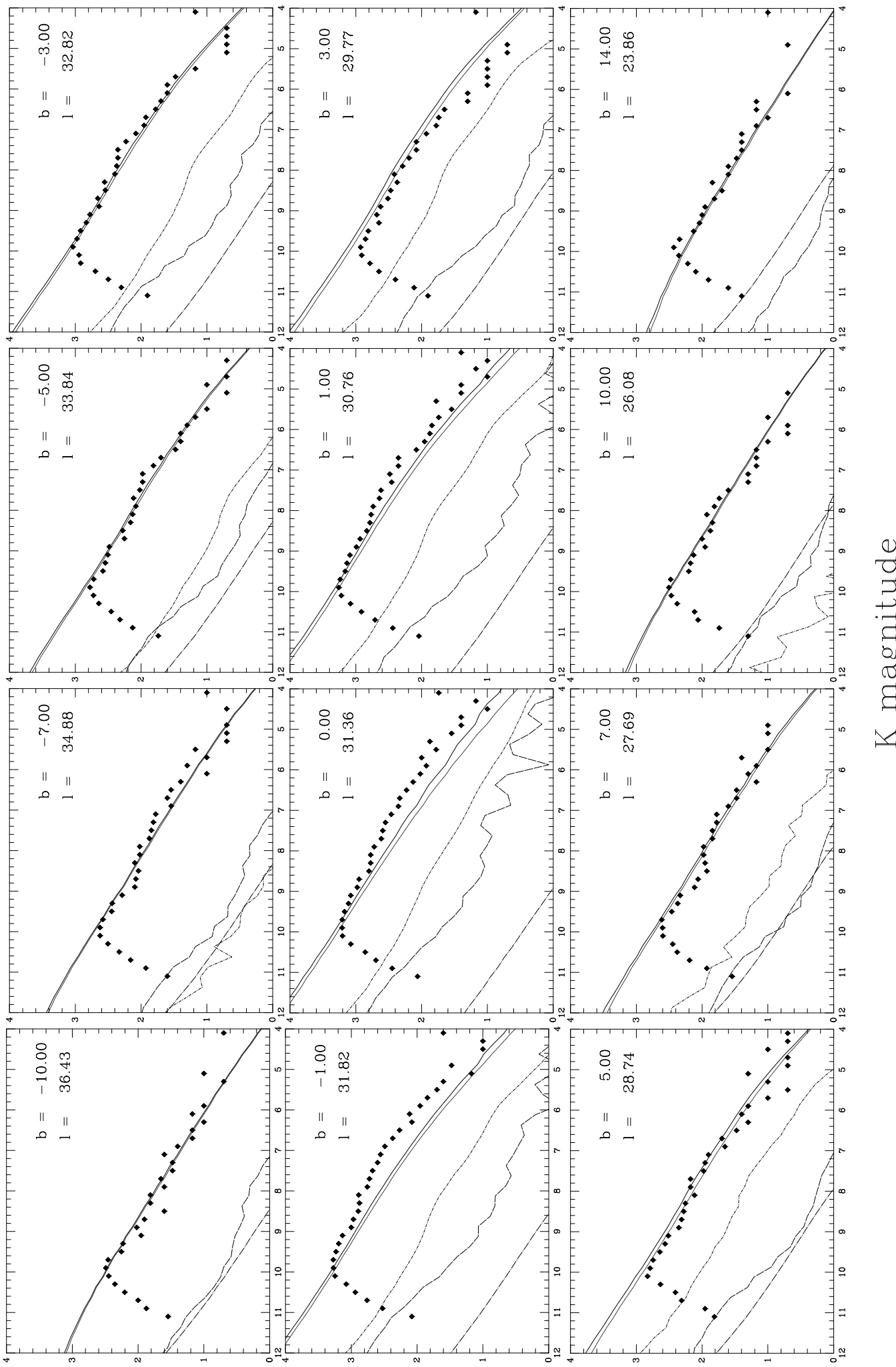

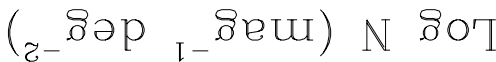

Figure A5: TMGS differential star counts (diamonds) plotted on top of the SKY predictions for the strip crossing the plane at $l=31^{\circ}$. 


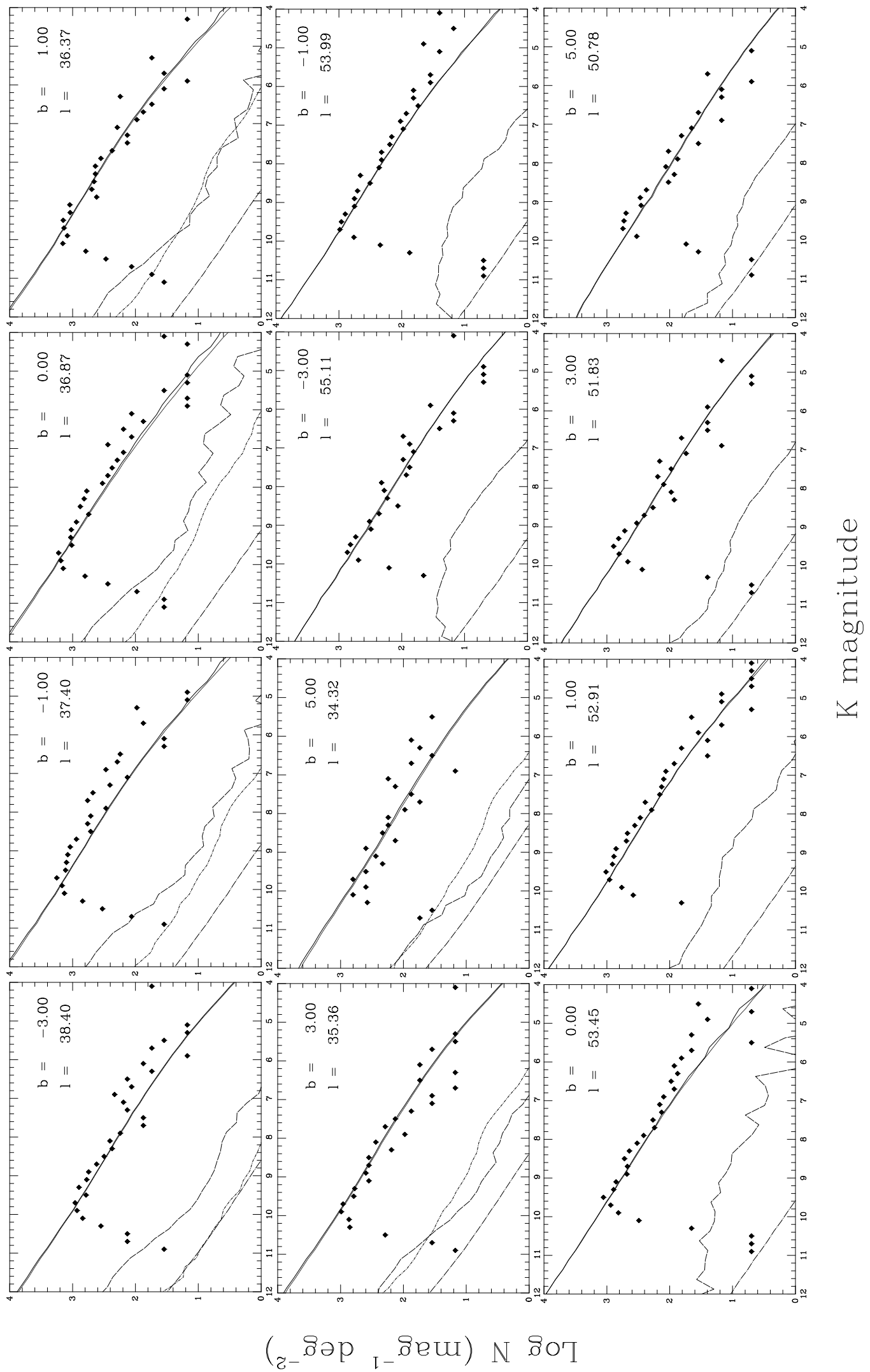

Figure A6: TMGS differential star counts (diamonds) plotted on top of the SKY predictions for the strips crossing the plane at $l=37^{\circ}$ and $l=53^{\circ}$. 

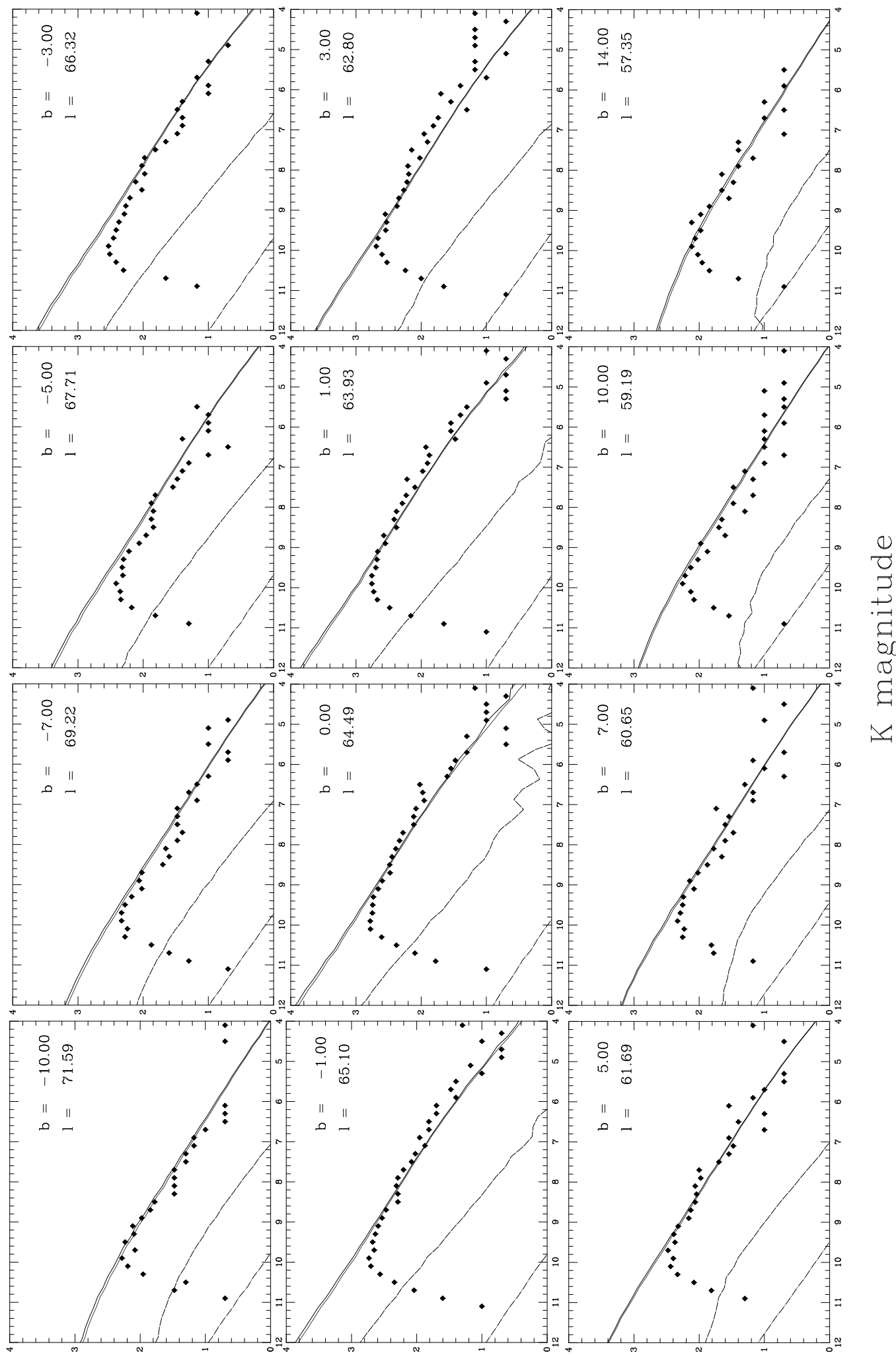

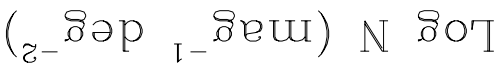

Figure A7: TMGS differential star counts (diamonds) plotted on top of the SKY predictions for the strip crossing the plane at $l=65^{\circ}$. 

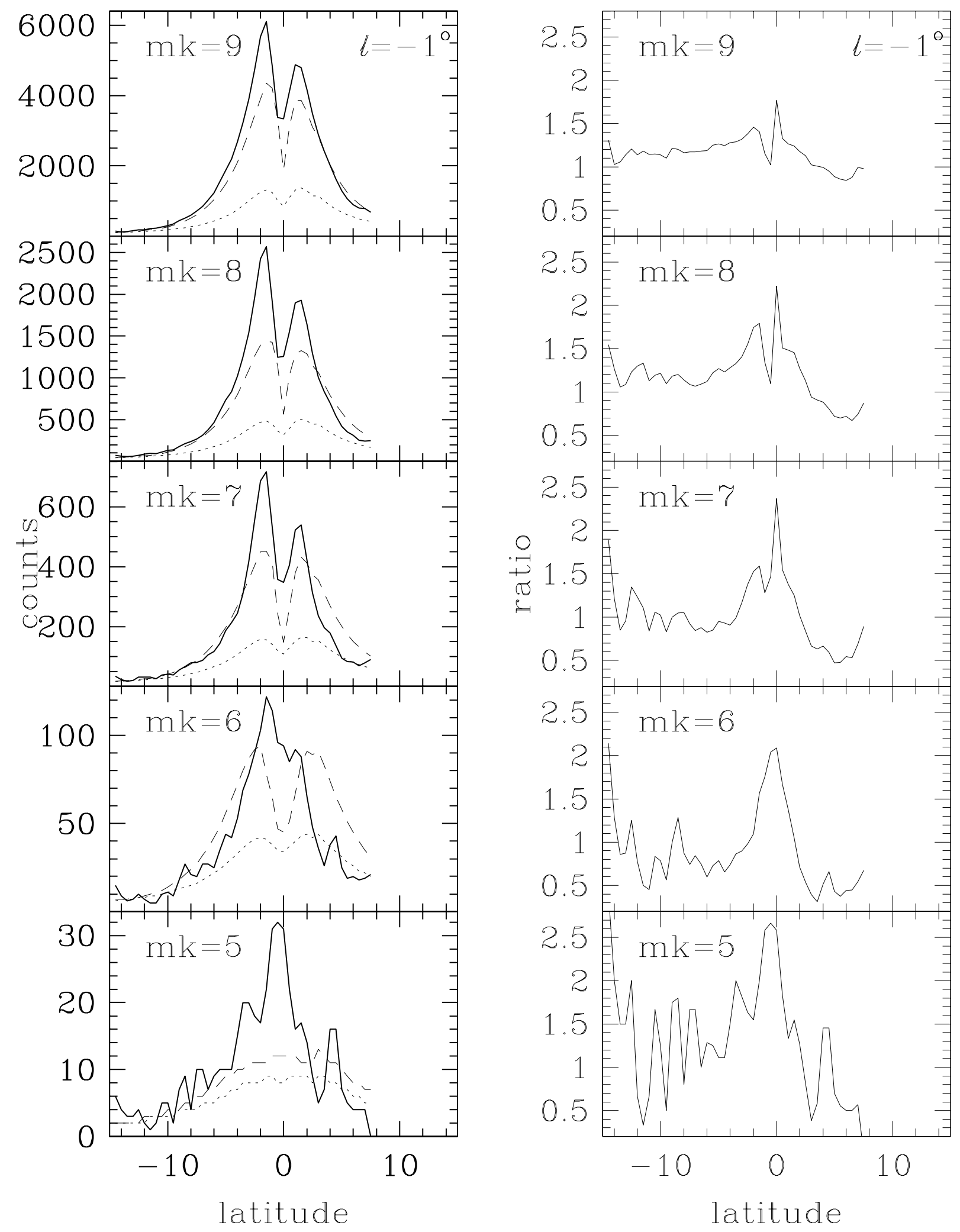

Figure B1: Right: cumulative star counts; TMGS (solid line); SKY total (dashed); SKY disc (dotted). Left: Ratio of TMGS to SKY total 

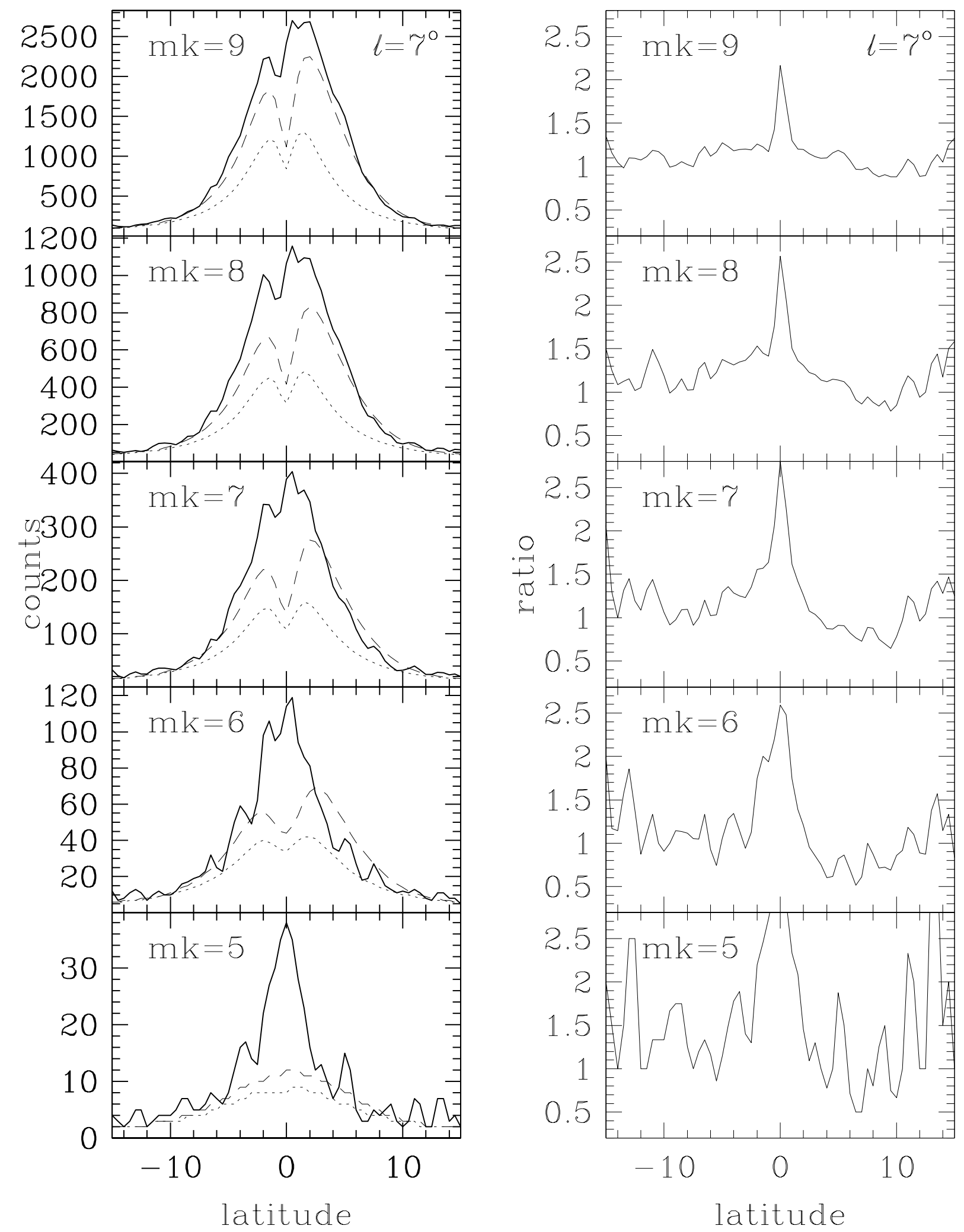

Figure B2: Right: cumulative star counts; TMGS (solid line); SKY total (dashed); SKY disc (dotted). Left: Ratio of TMGS to SKY total 

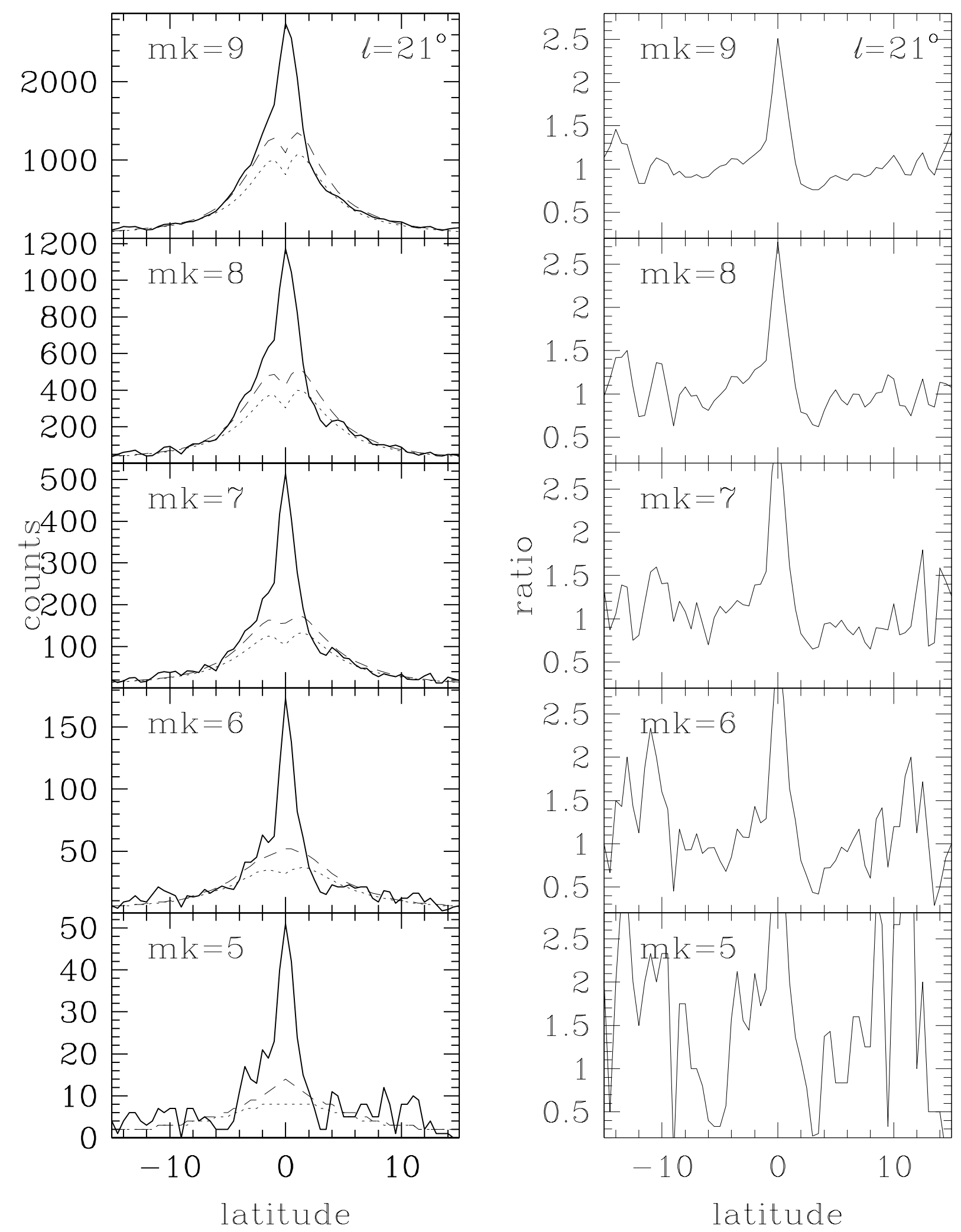

Figure B3: Right: cumulative star counts; TMGS (solid line); SKY total (dashed); SKY disc (dotted). Left: Ratio of TMGS to SKY total 

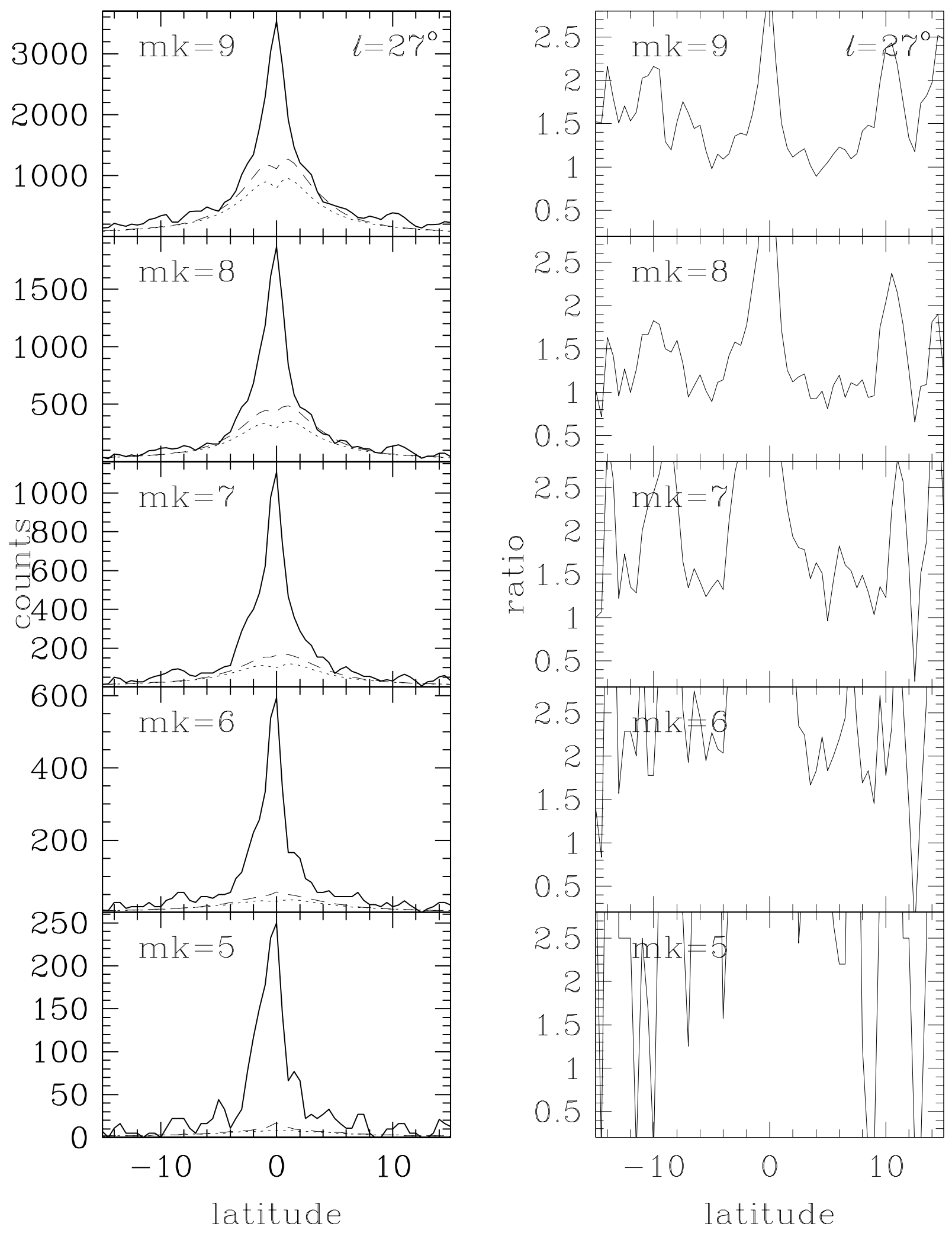

Figure B4: Right: cumulative star counts; TMGS (solid line); SKY total (dashed); SKY disc (dotted). Left: Ratio of TMGS to SKY total 

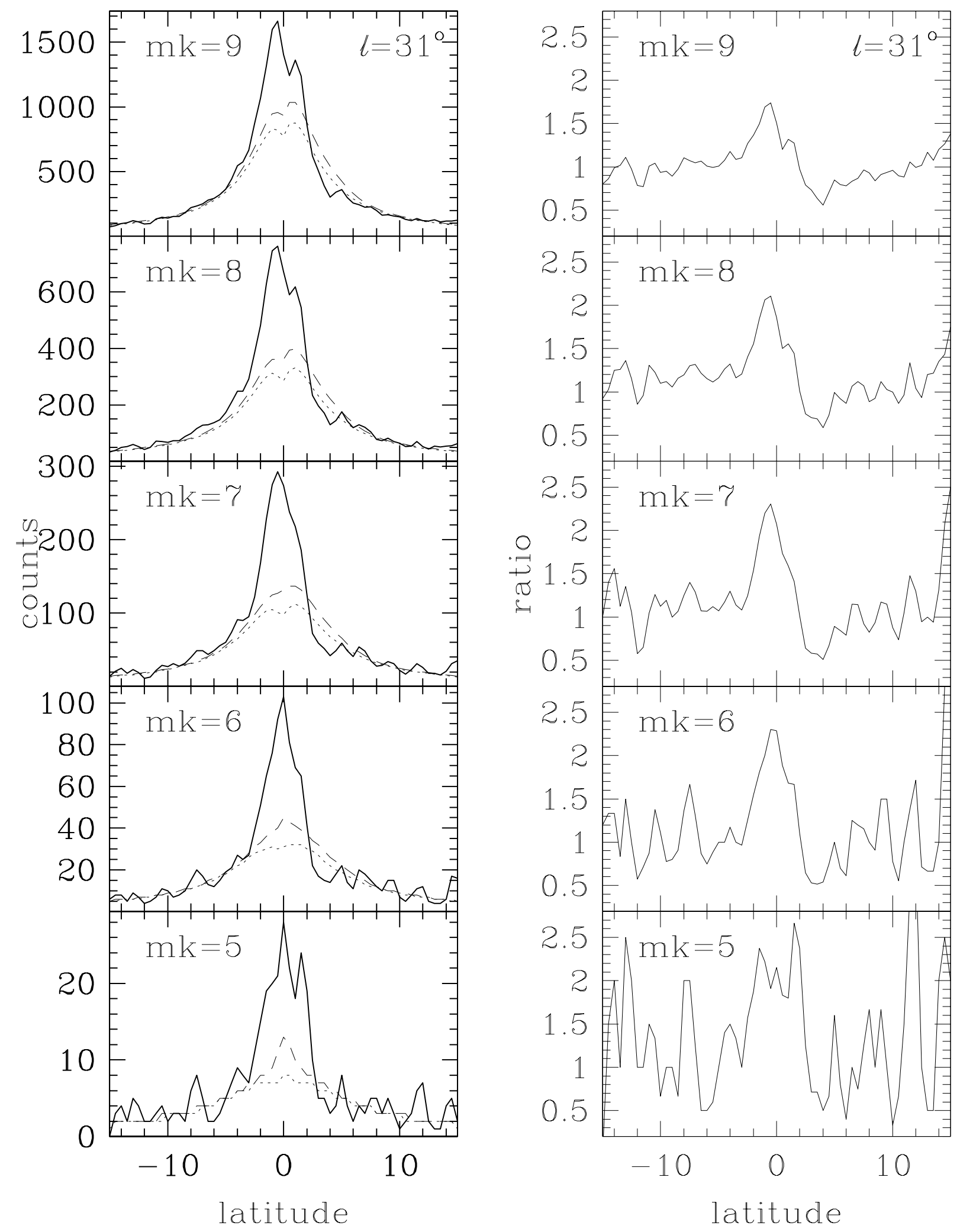

Figure B5: Right: cumulative star counts; TMGS (solid line); SKY total (dashed); SKY disc (dotted). Left: Ratio of TMGS to SKY total 

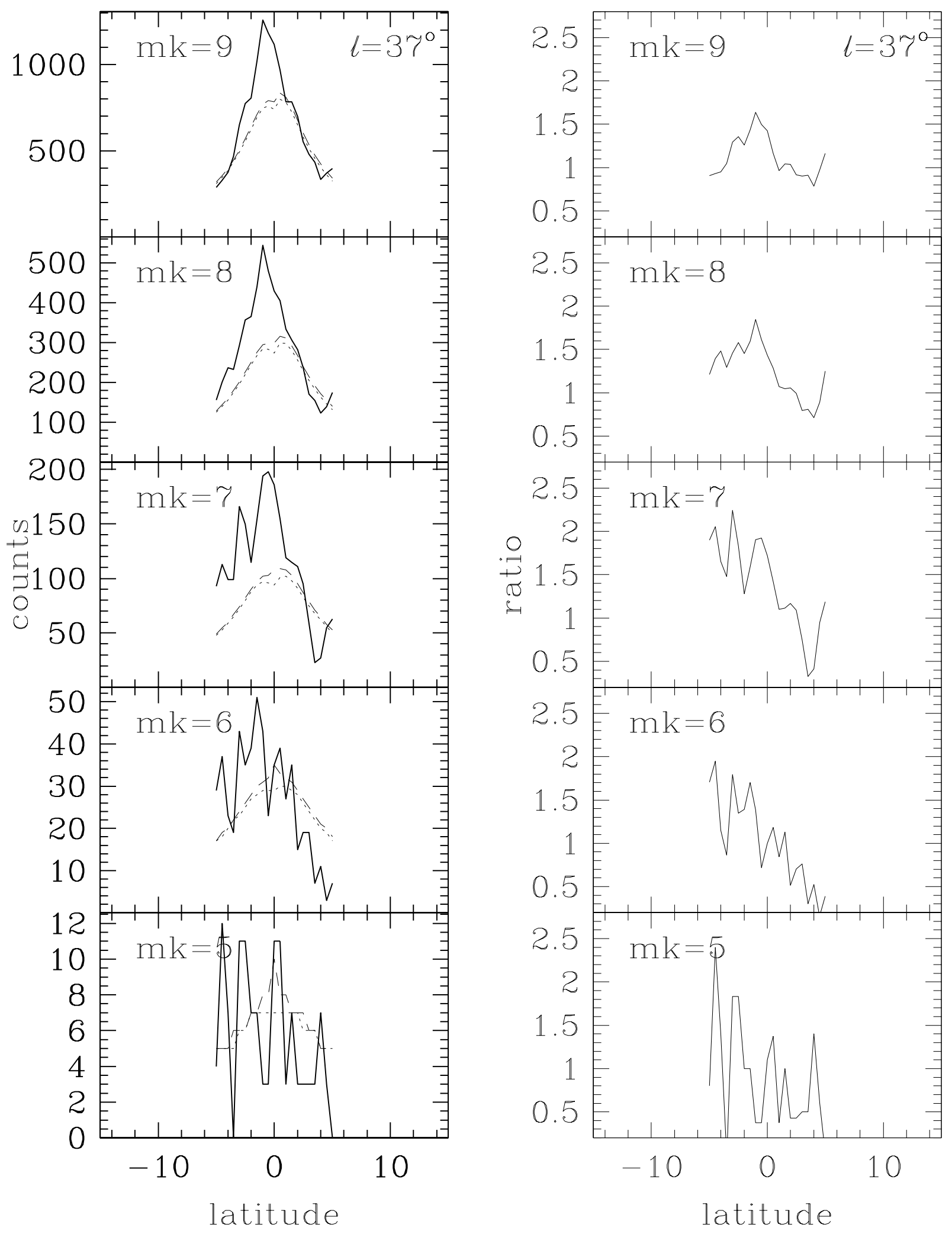

Figure B6: Right: cumulative star counts; TMGS (solid line); SKY total (dashed); SKY disc (dotted). Left: Ratio of TMGS to SKY total 

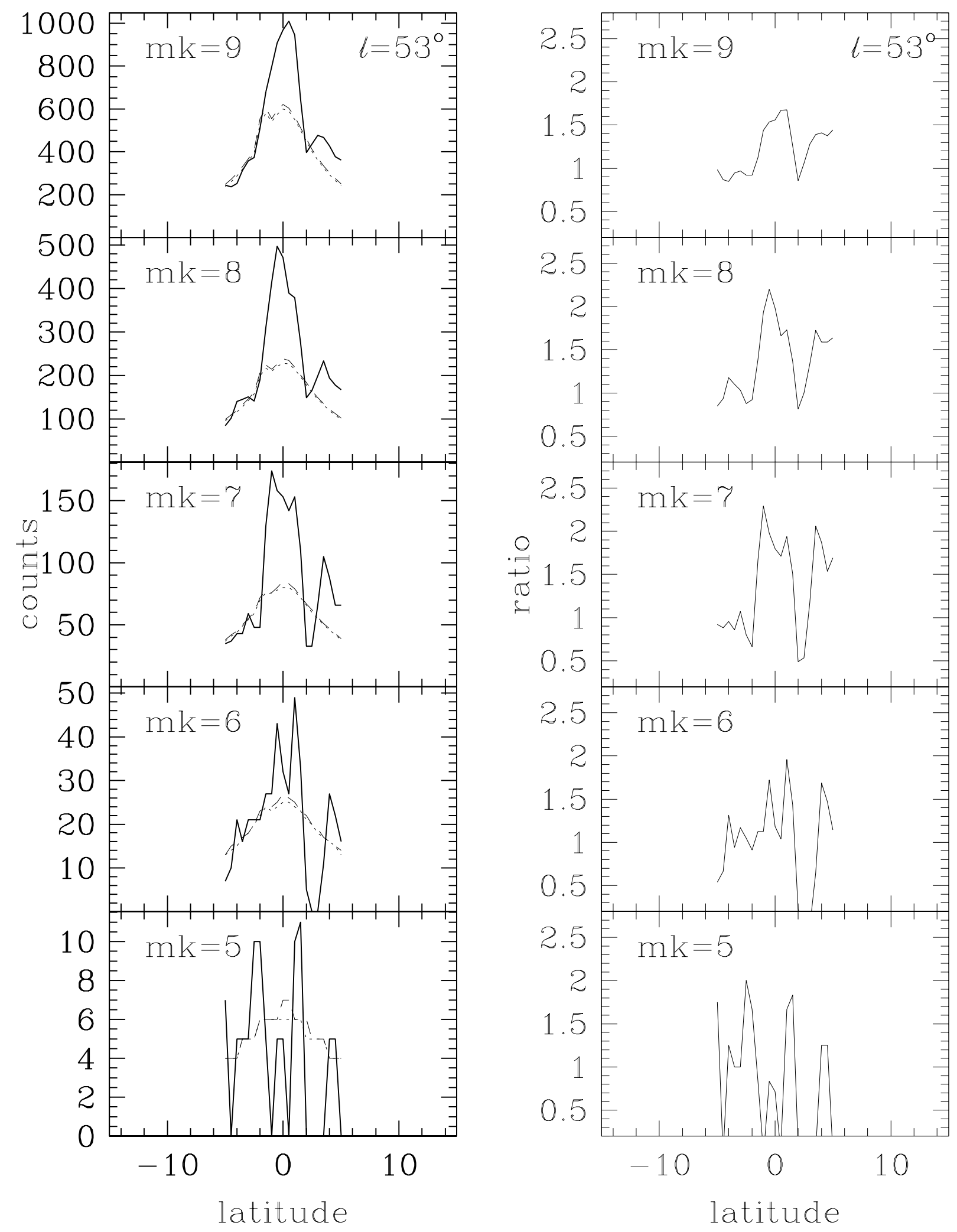

Figure B7 Right: cumulative star counts; TMGS (solid line); SKY total (dashed); SKY disc (dotted). Left: Ratio of TMGS to SKY total 

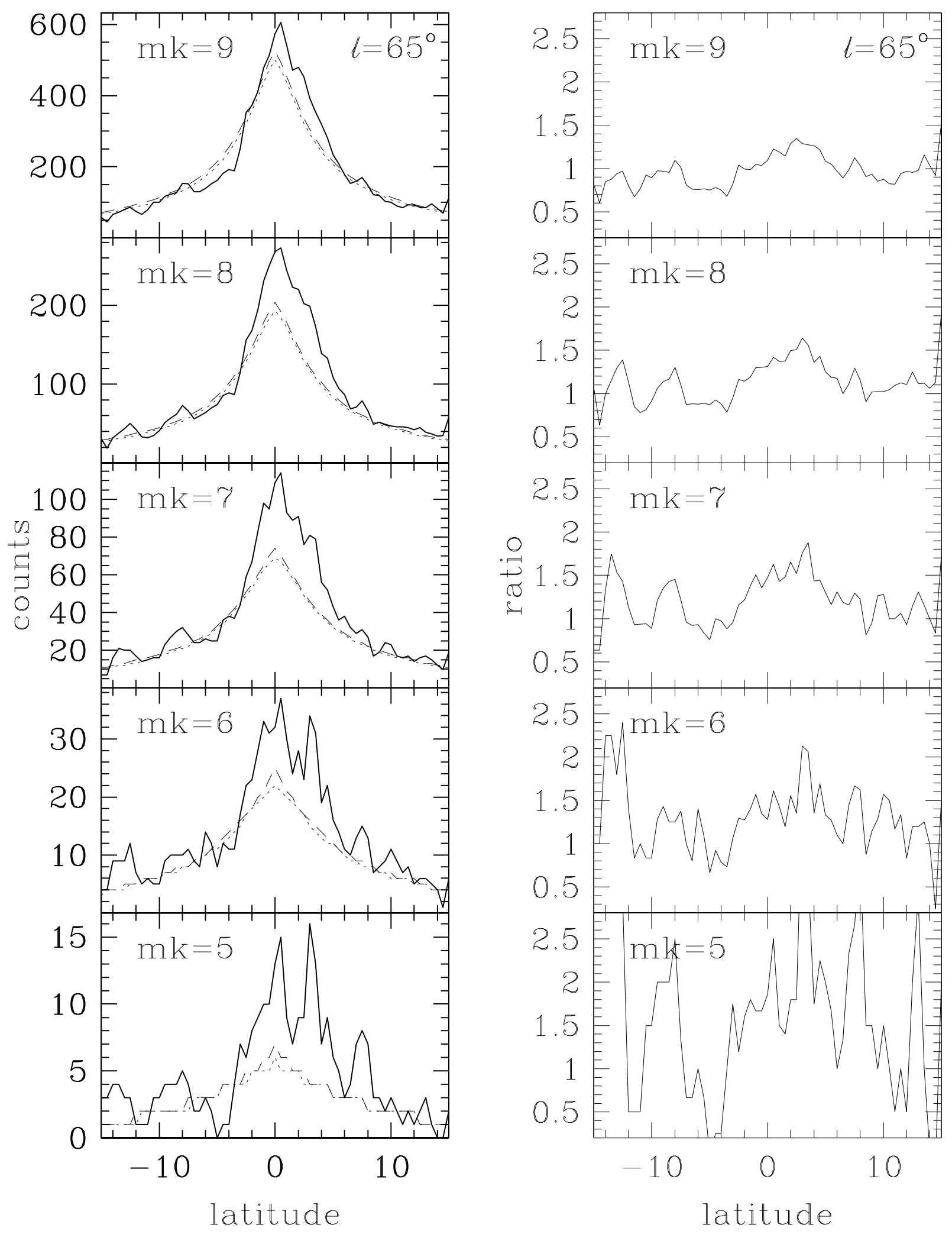

Figure B8 Right: cumulative star counts; TMGS (solid line); SKY total (dashed); SKY disc (dotted). Left: Ratio of TMGS to SKY total 

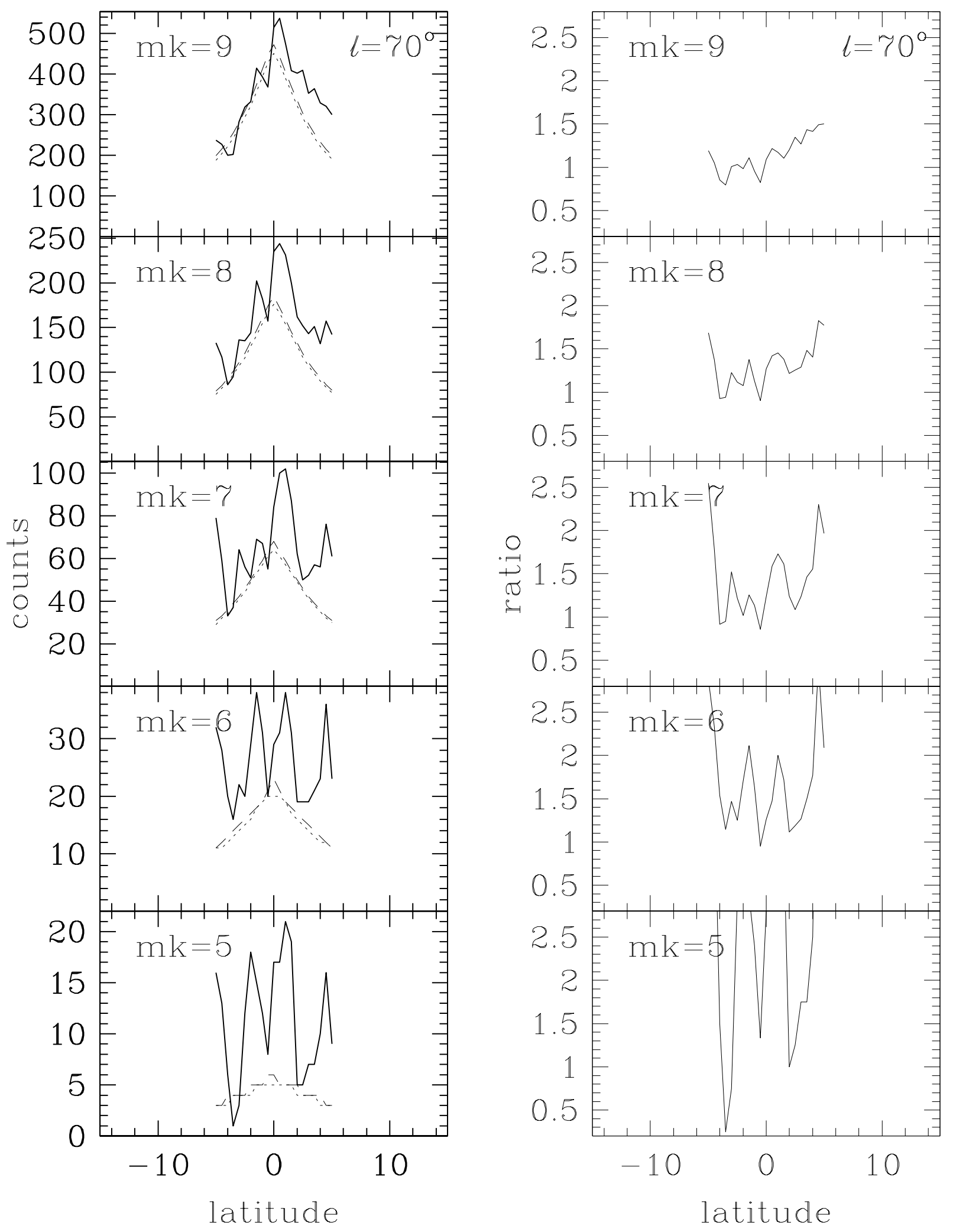

Figure B9 Right: cumulative star counts; TMGS (solid line); SKY total (dashed); SKY disc (dotted). Left: Ratio of TMGS to SKY total 

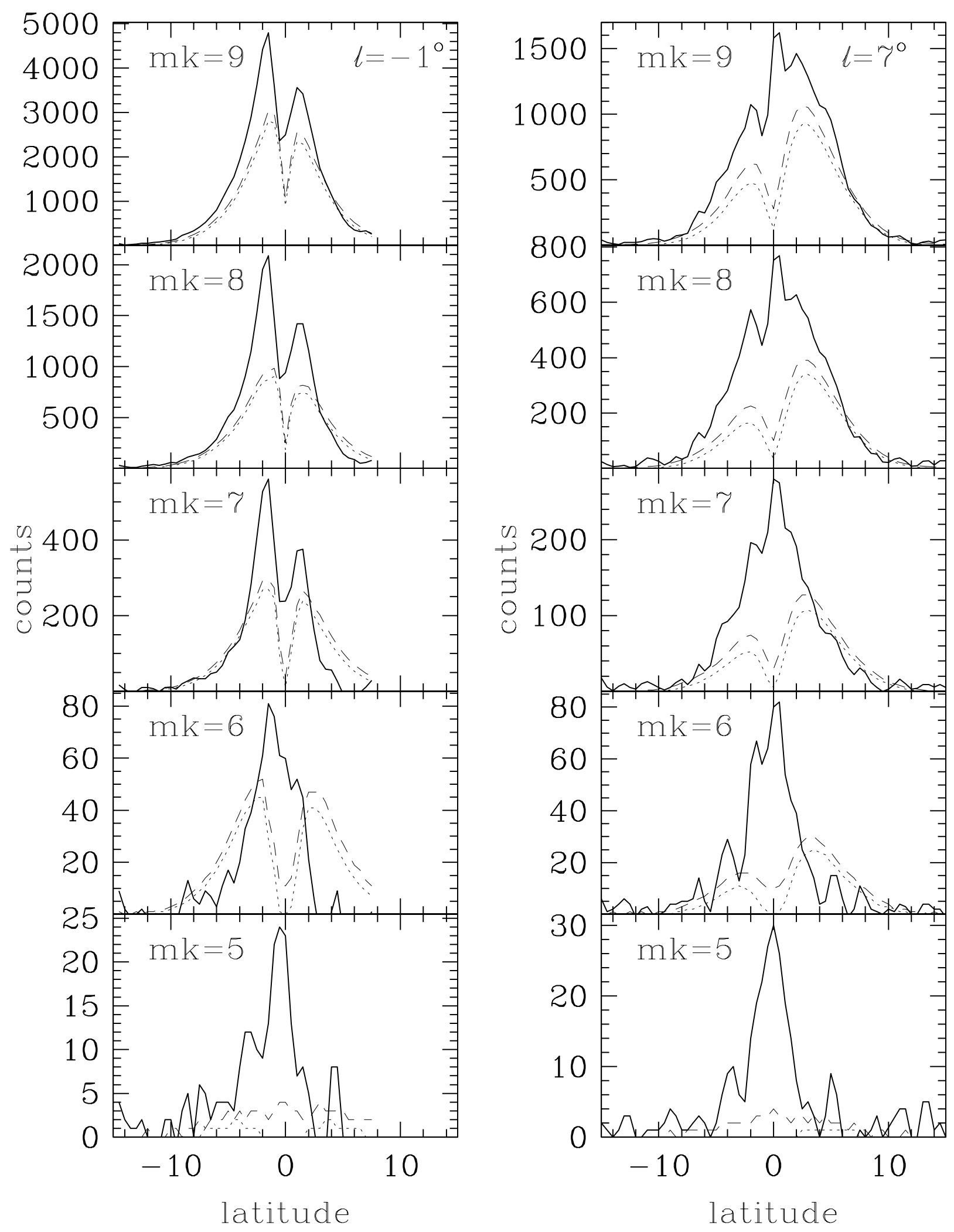

Figure C1\& C2 TMGS - SKY disc (solid); SKY total - SKY disc (dashed); SKY bulge (dotted) 

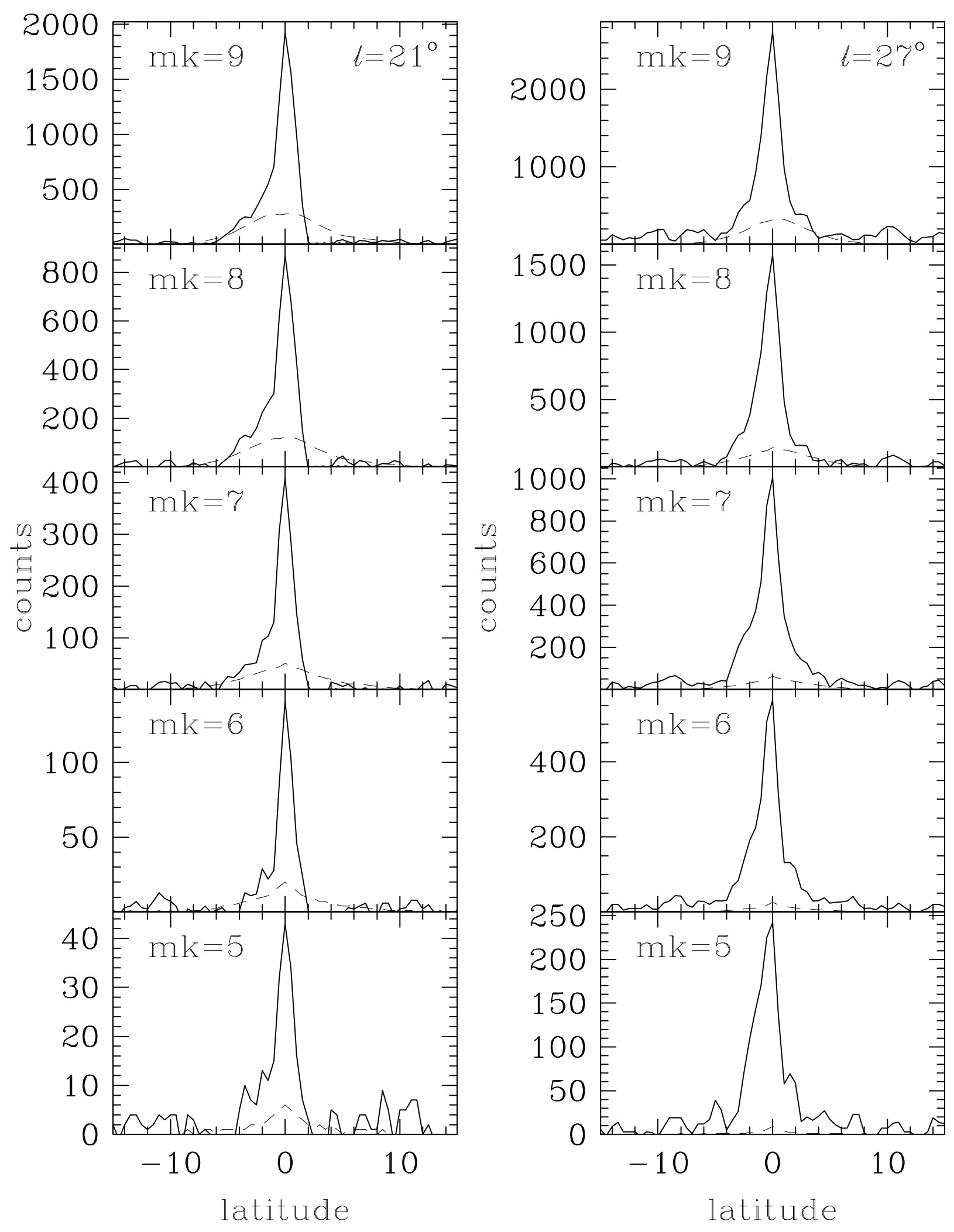

Figure C3 \& C4 TMGS - SKY disc (solid); SKY total - SKY disc (dashed); SKY bulge (dotted) 

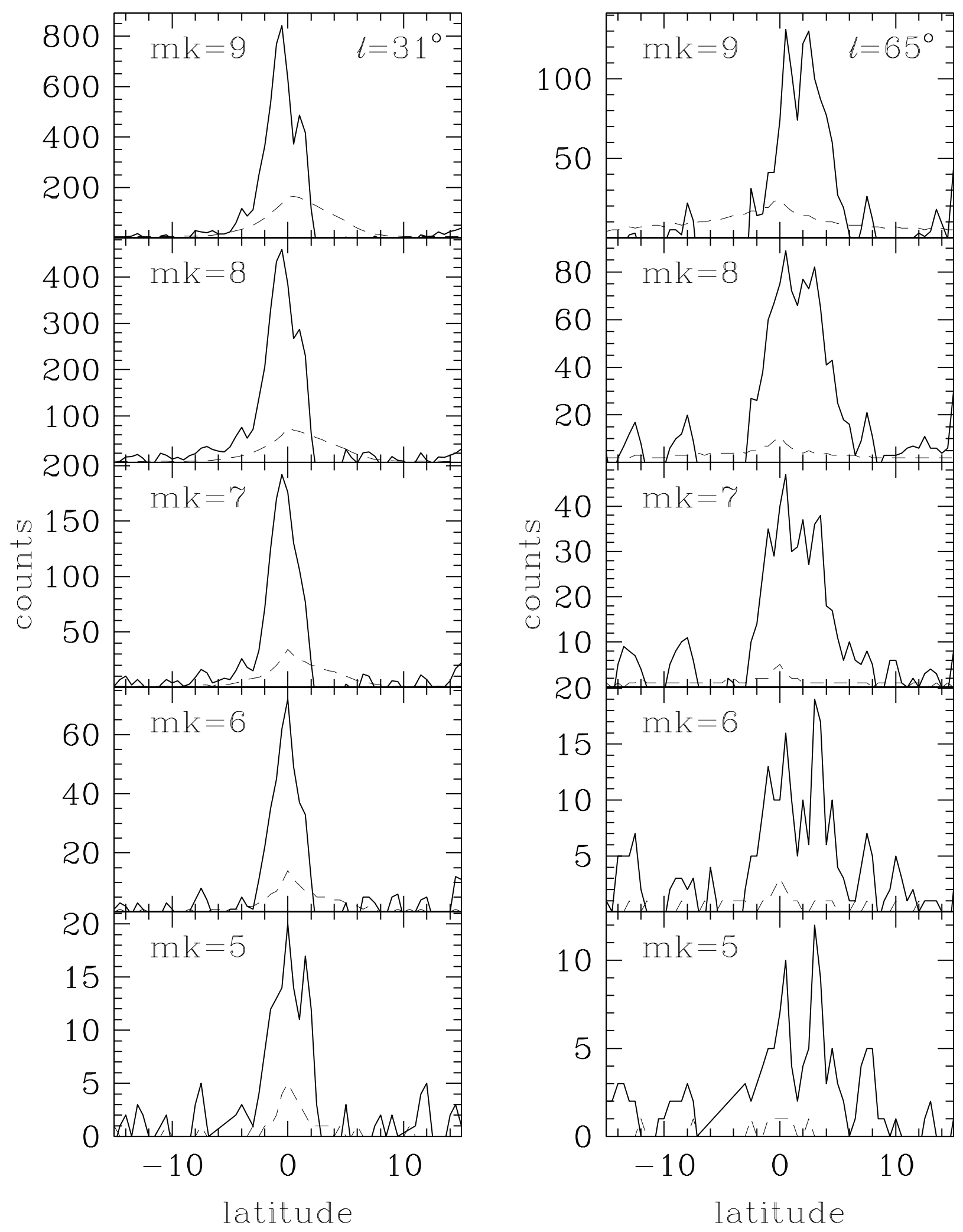

Figure C5 \& C6 TMGS - SKY disc (solid); SKY total - SKY disc (dashed); SKY bulge (dotted) 